\title{
PLIF Visualization of Active Control of Hypersonic Boundary Layers Using Blowing
}

\author{
Brett F. Bathel* \\ University of Virginia, Charlottesville, VA, 22904 \\ Paul M. Danehy ${ }^{\dagger}$, Jennifer A. Inman ${ }^{\ddagger}$, David W. Alderfer ${ }^{\S}$, and Scott A. Berry ${ }^{* *}$ \\ NASA Langley Research Center, Hampton, VA, 23681-2199
}

\begin{abstract}
Planar laser-induced fluorescence (PLIF) imaging was used to visualize the boundary layer flow on a 1/3-scale Hyper-X forebody model. The boundary layer was perturbed by blowing out of orifices normal to the model surface. Two blowing orifice configurations were used: a spanwise row of 17 -holes spaced at $1 / 8$ inch, with diameters of 0.020 inches and a single-hole orifice with a diameter of 0.010 inches. The purpose of the study was to visualize and identify laminar and turbulent structures in the boundary layer and to make comparisons with previous phosphor thermography measurements of surface heating. Jet penetration and its influence on the boundary layer development was also examined as was the effect of a compression corner on downstream boundary layer transition. Based upon the acquired PLIF images, it was determined that global surface heating measurements obtained using the phosphor thermography technique provide an incomplete indicator of transitional and turbulent behavior of the corresponding boundary layer flow. Additionally, the PLIF images show a significant contribution towards transition from instabilities originating from the underexpanded jets. For this experiment, a nitric oxide/nitrogen mixture was seeded through the orifices, with nitric oxide (NO) serving as the fluorescing gas. The experiment was performed in the 31-inch Mach 10 Air Tunnel at NASA Langley Research Center.
\end{abstract}

\section{Introduction}

$\mathrm{T}$

HE successful flight tests of the Hyper-X in 2004, both at Mach 7 and 10, demonstrated the capabilities of firstgeneration hypersonic technologies for scramjet-powered vehicles. The final vehicle configuration was based on concepts that were refined throughout the development of the Hyper-X program. A boundary layer control system, employed upstream of the engine inlet, was one such concept. The purpose of such a system is to condition the boundary layer flow, producing a controlled transition to turbulence prior to the scramjet inlet. For the Hyper-X flight tests, an array of discrete roughness elements were used to induce boundary layer transition. The application of blowing gas jets (active system), in addition to the passive method used on the Hyper-X, are the most effective methods to disturb, or trip, the boundary layer into transition and eventual turbulence. In addition to the characteristics of the flow field produced by such tripping elements, the resulting heat transfer profile along the vehicle is also of interest. While a transitional or turbulent flow condition is desired prior to the engine inlet in order to facilitate fuel/air mixing and combustion, the energy transferred from the gas to the vehicle surface is of concern from a materials and cooling perspective.

Wind tunnel testing of scaled Hyper-X models employing passive, discrete roughness trip elements has been performed at NASA's Langley Research Center in both the 20 -inch Mach 6 and 31-inch Mach 10 wind tunnels. ${ }^{1-5}$ The purpose of the tests was to measure the heat transfer profiles along the vehicle surface immediately downstream

\footnotetext{
${ }^{*}$ National Institute of Aerospace Graduate Student, Department of Mechanical and Aerospace Engineering, AIAA Student Member.

${ }^{\dagger}$ Research Scientist, Advanced Sensing and Optical Measurement Branch, MS 493, AIAA Associate Fellow.

* Research Scientist, Advanced Sensing and Optical Measurement Branch, MS 493, AIAA Member.

${ }^{\S}$ Research Scientist, Advanced Sensing and Optical Measurement Branch, MS 493.

** Research Scientist, Aerothermodynamics Branch, MS 408A, AIAA Senior Member.
} 
of the tripping elements, visualize flow patterns produced along the vehicle surface in this region, and to visualize shock wave generation and interaction. Measurements of the global surface heat transfer profiles were made along the underbody compression surfaces using the phosphor thermography method ${ }^{6}$. A set of four plates installed along the length of the Hyper-X model underbody and, treated with a temperature-sensitive phosphor coating, provided a visual representation and quantitative measurement of the magnitude of heat transfer. These flat plates were integrated into the model body along the compression ramps. Oil flow aided in characterizing flow patterns along the vehicle underbody and, more specifically, about the trip elements and along the compression surfaces. Schlieren imaging was used to visualize shock generation and interaction.

Previously, fundamental experiments involving blowing boundary layer trip devices and a flat plate model were performed in Langley's 20-inch Mach 6 and 20-inch Mach 8.5 tunnels. ${ }^{7}$ These experiments detailed the jet structure and corresponding downstream pressures, heat transfer profiles, and flow transitional locations. Experiments implementing blowing trips integrated onto a Hyper-X forebody model were performed by Berry et al. ${ }^{2}$ in Langley's 20-inch Mach 6 and 31-inch Mach 10 tunnel. Some of the results obtained in the 31-inch Mach 10 tunnel are further analyzed and characterized in this paper.

The heat transfer measurement and oil flow visualization techniques employed in previous Hyper-X model testing have provided detailed characterization of surface conditions. Visualization of the off-body flow field through the application of Schlieren imaging provided a description of shock generation and interaction. However, limitations existed in providing detailed descriptions of the off-body flow field. While schlieren imaging can provide path-averaged visualization of high density gradient regions, like shock waves, regions of low slowly varying density, such as those within the boundary layer, cannot be studied in detail. Since schlieren imaging is path averaged, this method is unsuitable for measuring small-scale flow structures associated with transitional and turbulent flow. Therefore, to compliment these techniques and provide detailed measurement of flow structures within the boundary layer itself, a non-intrusive imaging technique, planar laser-induced fluorescence (PLIF), has been employed.

This paper describes work utilizing PLIF to image boundary layer flow characteristics produced by blowing trips integrated onto a $1 / 3$-scale Hyper-X forebody wind tunnel model. The majority of test conditions presented in this paper are nearly identical to several free-stream conditions examined in prior work by Berry et al. ${ }^{2}$ performed in the same facility and compliments work performed by Danehy et al. ${ }^{8}$ using stereoscopic imaging which provided visualization of the boundary layer flow with greater depth. This work specifically examines two blowing trip configurations have been examined at eight primary jet flow rates. The PLIF image results, taken at various locations throughout the boundary layer along the first and second compression ramps, are also compared with phosphor thermography images obtained in prior testing.

\section{Experimental Setup and Analysis}

The test apparatus consisted of three main components: the test articles, the wind tunnel facility and the PLIF system. The analysis involved image processing and rendering in a three-dimensional software environment. These are summarized briefly in this section with references describing where more details can be found.

\section{A. Test Article}

A 1/3-scale Hyper-X forebody model was fabricated primarily of aluminum. The leading edge of the model consisted of a detachable leading edge (with a nose radius of 0.010 inches) made of stainless steel. Because the flow region of interest was along the compression surface on the bottom side of the model, the lateral edges extending beyond 5 inches from the

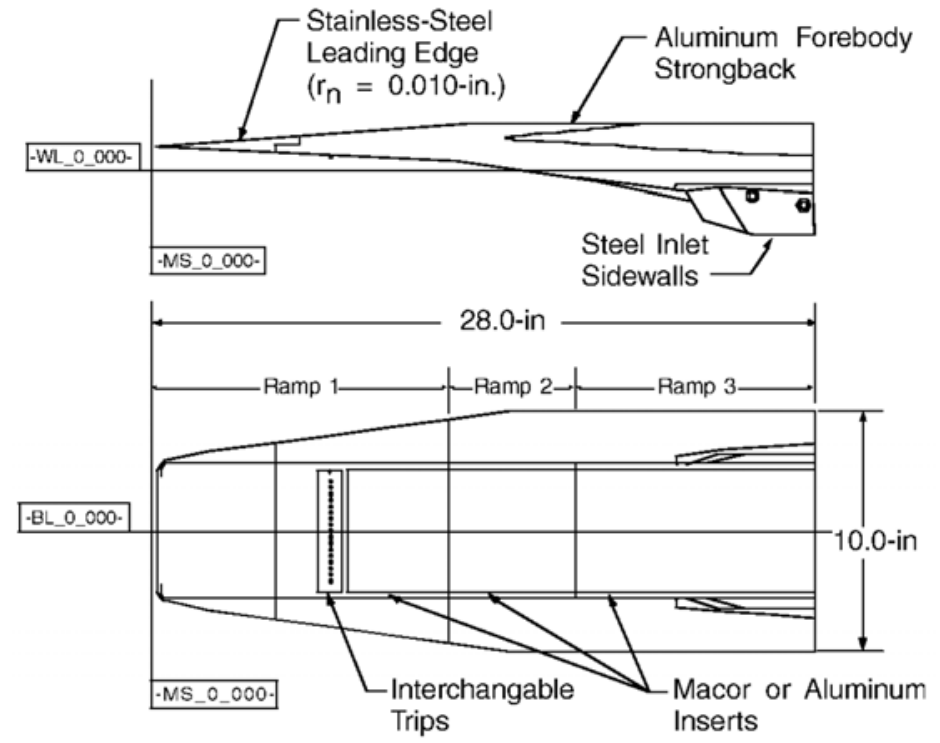

Figure 1. Schematic of Hyper-X forebody model (from Ref. 2). 
centerline were truncated to reduce the overall model profile within the tunnel. The main body of the model had a single cavity located 7.418 inches from the leading edge, with a depth of 0.5 inches into the model, a spanwise width of 5.079 inches, and a streamwise length of 1 inch. The cavity allowed for interchanging of the blowing trips and accommodation of plumbing, which supplied a blowing gas mixture from an external source. An additional cavity, located 0.25 inches beyond the trip cavity and with the same width and depth, extended the length of the model underbody and accommodated either four Macor or four aluminum plates. These served as the angled compression surfaces. For this experiment, only the flow fields along the first and second compression ramps were studied. Figure 1 shows a general schematic of the forebody model.

Each blowing trip insert consisted of a rectangular 5-by-1inch plate with a cavity in the bottom surface to allow for attachment of a plenum and connection of plumbing to provide the pressurized gas for blowing. The two configurations consisted of the following: (1) a row of 17, 1/8-inch spaced, 0.020 -inch diameter holes and (2) a single 0.010-inch diameter hole, with each configuration being laterally and longitudinally symmetric at the surface. Figure 2 shows a computer rendering of the top surface of each blowing trip configuration, with the size of the holes shown at twice the diameter of the original size for visibility.

A mixture of nitric oxide (NO) gas and diatomic nitrogen $\left(\mathrm{N}_{2}\right)$ was supplied to the plenum via a system of four mass flow controllers. Two Teledyne-Hastings mass flow controllers metered the NO supply, an HFC-202B and HFC-302, with maximum capacities of 100 standard cubic-centimeters per minute $(\mathrm{sccm})$ and 1000 sccm, respectively. To control the $\mathrm{N}_{2}$ gas, an HFC-302, an HFC-202, and an HFC-203A, were used to supply gas at maximum flow rates of $1000 \mathrm{sccm}, 3$ standard liters per minute (slpm), and $51 \mathrm{slpm}$, respectively. The plumbing system that supplied gas to the mass flow controllers and ultimately the plenum itself was

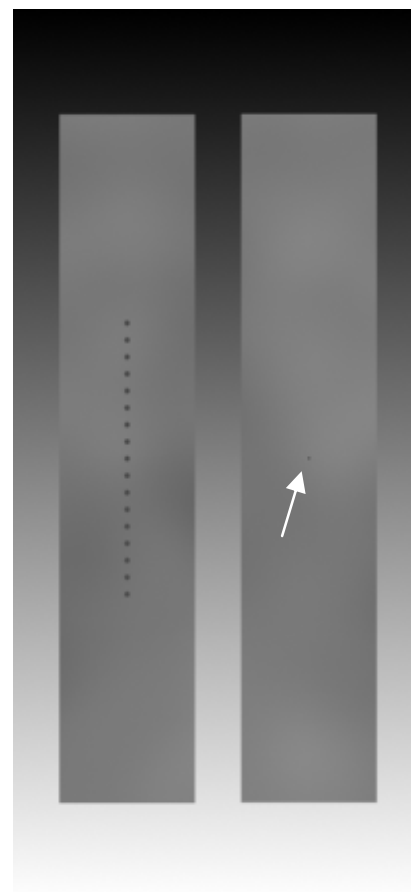

Figure 2. Blowing trip inserts with two hole configurations. designed with a combination of valves such that any two controllers could be used to separately provide the NO and $\mathrm{N}_{2}$ gas streams at a given plenum/free stream pressure ratio. An Endevco piezoresistive pressure transducer (0-15 psia range) and a Type-E thermocouple were used to monitor conditions in the plenum so that the controllers could be adjusted provide the appropriate plenum pressure condition and blowing flow rate (to match conditions in Ref. 2). Table 1 provides the run number (for imaging performed on the first and second compression ramps), target plenum pressures, and target flow rates tested for this paper. In each case, however, the plenum pressures and flow rates usually fell slightly below (in the case of the 17-hole configuration) or varied about (in the case of the single-hold configuration) the target points.

Table 1. Target test conditions.

\begin{tabular}{|c|c|c|c|c|c|c|c|}
\hline \multicolumn{2}{|c|}{ Single Row, 17 Hole (0.02 in diameter) Blowing Trip } & \multicolumn{3}{c|}{ Single Hole (0.01 inch diameter) Blowing Trip } \\
\hline \multicolumn{2}{|c|}{ Runs } & \multirow{2}{*}{ Pressure (psi) } & \multirow{2}{*}{ Flow Rate (slpm) } & \multicolumn{2}{|c|}{ Runs } & \multirow{2}{*}{ Pressure (psi) } & \multirow{2}{*}{ Flow Rate (slpm) } \\
\cline { 1 - 5 } Current & Ref. 2 & & Current & Ref. 2 & & \\
\hline 6,14 & 71 & 0.4 & 0.69 & 22,18 & 30 & 3.8 & 0.11 \\
\hline 7,13 & 83 & 1.6 & 3.54 & 21,17 & 32 & 17.4 & 0.61 \\
\hline 4,12 & 81 & 6.5 & 15.3 & 23,19 & - & - & 1.8 \\
\hline 5,15 & 80 & 12.8 & 31.6 & & & & \\
\hline 3,16 & 79 & 19.9 & 49.7 & & & \\
\hline
\end{tabular}

Variation of the flowrate (or pressure) supplied to the plenum produced laminar, transitional, and fully turbulent boundary layers, which were observed directly downstream of the trips. To acquire the images, two CCD cameras were mounted side-by-side, facing approximately normal to the vehicle surface. One camera, fitted with a $105 \mathrm{~mm}$ focal length lens, provided a nearly three times higher-resolution near-field image of the flowfield directly downstream of the trip. The other camera, fitted with a $45 \mathrm{~mm}$ focal length Cerco lens, provided a retracted view of the flowfield with a larger field of view. 


\section{B. Test Facility}

The 31-Inch Mach 10 Air Tunnel is an electrically-heated blowdown facility located at NASA Langley Research Center in Hampton, Virginia, USA. Reference 9 details this facility, a brief summary of which is provided here. The facility has a nominal Mach number of 10 and a 31-inch square test section and operates on electrically heated, compressed air. Large windows, transparent in the ultraviolet down to approximately $190 \mathrm{~nm}$, form three walls (including top, side and bottom) of the test section with the fourth wall formed by the model injection system. The model is side-mounted to this fourth wall. Run durations for the current tests were about one minute. Two different facility stagnation pressures, $\mathrm{P}_{0}$, were investigated: $9.31 \mathrm{MPa}(1350 \mathrm{psia})$ and $10.0 \mathrm{MPa}(1450 \mathrm{psia})$. The two operating pressures simulate freestream unit Reynolds numbers of 1.85 million per foot and 1.96 million per foot for the 17-hole and single hole trip configurations, respectively. The nominal stagnation temperature was $1,000 \mathrm{~K}$ $\left(1,800^{\circ}\right.$ Rankine $)$ for the experiment described herein.

\section{Planar-Laser Induced Fluorescence (PLIF) System and Image Processing Techniques}

The PLIF system consisted primarily of the laser system, sheet-forming optics and the detection system. The laser system had a Spectra-Physics Pro-230-10 Nd:YAG laser operating at $10 \mathrm{~Hz}$, a Spectra-Physics PDL-2 tunable pulsed dye laser, and a wavelength extender. The laser output was tuned to a wavelength of $226.256 \mathrm{~nm}$, chosen to excite the strongly fluorescing spectral lines of NO near the $\mathrm{Q}_{1}$ branch head. Lenses formed the laser beam into a sheet and an intensified CCD camera acquired images. Flow visualization images were acquired at $10 \mathrm{~Hz}$ with a $1 \mu \mathrm{s}$ camera gate and a spatial resolution of about 270-285 pixels/inch for the near-field imaging and about 90-95 pixels/inch for the far-field imaging, depending on the camera configuration. A printed matrix of equally spaced squares affixed to the model (referred to as dotcard) was captured with each camera at various model injection locations. These images were used to correct for image distortions using a method described in Ref. 10 and to determine image magnification in post-processing. To acquire planar images at several off-body locations, a scanning laser sheet with a scanning profile similar to that in Figure 3, was adopted for each run. The thickness of the laser sheet was measured to be 0.03 inches at the series of tests on the 17-hole injection and the first make or plate. For all of the other runs a thicker laser sheet was used with a thickness of approximately 0.09 inches. Further details of the PLIF system and its use in the 31-Inch Mach 10 Air Tunnel can be found in Refs. 11, 12, and 13 .

Over the course of testing, it was determined that variations in laser sheet intensity required that image corrections be performed on a shot-by-shot basis. All image processing was performed using MATLAB ${ }^{\circledR}$. For the images with a wider ( $\sim 5.5$ in. $x 5.5$ in.) field of view, a region encompassing the primary flow features was averaged along the direction of laser propagation and the entire image divided by this laser profile. For the 17-hole trip configuration, a constant was subtracted from each image to provide for background correction.

For the single-hole configuration, greater signal intensity variation was observed in the stream-wise direction, which limited the effectiveness of using the above method for obtaining an averaged laser profile and of using a constant background correction. Therefore, a low signal intensity region was selected and averaged row-by-row to provide a background profile. This profile was then smoothed using a moving average filter with a 100-pixel wide window and then subtracted, row-by-row, from each image. Once this background correction was made, the center part of the image immediately surrounding the gas jet was averaged, row-by-row, to produce an approximate measure of the laser sheet intensity profile. This profile was then smoothed with a moving average filter with a 50 to 100-pixel wide window. The entire image was then divided by this profile. The final images were again smoothed with a 1-pixel radius disk filter and a false color table applied.

American Institute of Aeronautics and Astronautics 


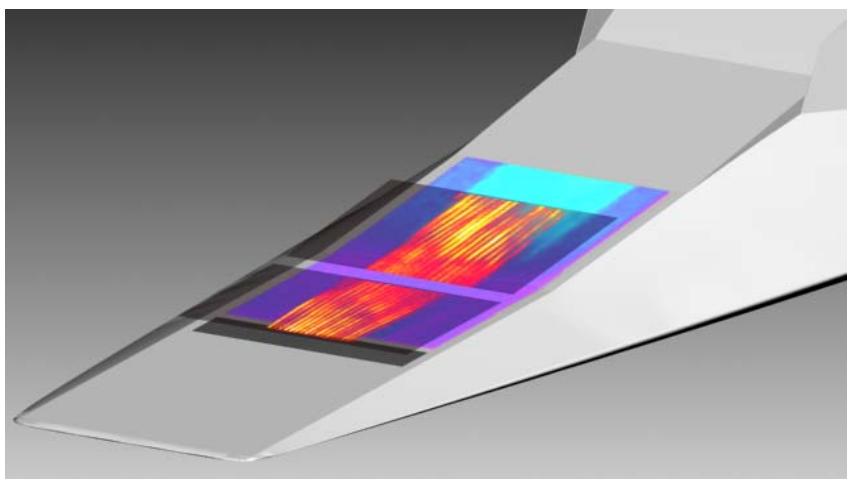

Figure 4. Perspective ViDI image for Runs 7 and 13.

\section{Virtual Diagnostics Interface (ViDI)}

The Virtual Diagnostics Interface (ViDI), described in Ref. 14, is a software tool, developed at NASA Langley Research Center, which provides unified data handling and interactive threedimensional display of experimental data and computational predictions. It is a combination of custom-developed software applications and Autodesk $^{\circledR} 3 \mathrm{ds} \operatorname{Max}^{\circledR}$, a commercially available, CAD-like software package for three-dimensional rendering and animation. Currently, ViDI technology is applied to three main areas: 1) pre-test planning and optimization; 2) visualization and analysis of experimental data and/or computational predictions; and 3) establishment of a central hub to visualize, store and retrieve experimental results. For this experiment, ViDI was used for post-test visualization of the PLIF data as in Ref. 15. All images presented in this paper represent flow conditions along the underbody of the $1 / 3$ scale Hyper-X forebody model. The phosphor-thermography images have been appropriately scaled and superimposed onto the first and second compression ramps. The PLIF images are displayed at various off-body locations corresponding to the laser sheet position recorded during testing. Figure 4 provides a perspective view of the ViDI technique.

\section{Results}

\section{A. 17-Hole Blowing Trip Configuration}

Figure 5 provides a summary comparison of PLIF measurements performed 0.075 inches off of the model surface with the corresponding phosphor-thermography images obtained in Ref. 2. Comparison between the phosphor thermography images and PLIF images will be discussed in the text associated with subsequent figures on a case-by-case basis. The images on the right side of the figure show the phosphor thermography data only. The images on the left side of the figure show the same phosphor thermography data with PLIF images superimposed. The color table for the phosphor thermography data represents the relative intensity of the ratio of heat-transfer coefficients based upon stagnation-point heating of a sphere examined in Ref. 16. The white arrows located along the upper portion of the phosphor-thermography images represent the point where the onset of transition was determined to have occurred, based upon methods described in Ref. 2. The color table applied to the PLIF images is representative of the PLIF fluorescence intensity which is proportional to the NO concentration. Additionally, the PLIF intensity is also sensitive to temperature, pressure, and velocity, although efforts have been made to suppress these sensitivities in the current experiment. The darkened rectangles indicate the primary field of view of the camera. In all cases, portions of the image not containing PLIF data have been truncated to allow a view of the underlying phosphor data. Note that images near the first compression ramp were acquired on separate runs from those near the second compression ramp, as these two locations represent separate imaging planes and required a repositioning of the laser sheet. Also note that the images in the figures have been rendered using a virtual camera, positioned normal to the flow axis of the wind tunnel, so both PLIF image planes appear at an angle. In all cases, the intersection of the first and second compression ramp is denoted by a white line drawn on the phosphorthermography images. While imaging the second compression ramp, the laser sheet was positioned to overlap portions of the first compression ramp. This overlap resulted in the laser sheet intersecting the flow along the end of the first compression ramp at an angle, which can be seen in all PLIF images showing flow over the second ramp. Due to this geometry, in near-surface visualizations on the second compression ramp the laser sheet intersects the surface of the model at the first compression ramp.

Figure 6 shows PLIF images of boundary layer flows, using a $\sim 3.6 \mathrm{slpm}$ total blowing flow rate, acquired in several off-body planes ( 0.075 inches, 0.1125 inches, and 0.15 inches away from the model surface) along the first and second compression ramps of the Hyper-X model. The corresponding phosphor-thermography image is displayed below the PLIF image, superimposed onto the vehicle surface. At each image plane location (Figure $6 \mathrm{~b}, \mathrm{c}, \mathrm{d})$, the individual jets emanating from the blowing trip insert maintain a consistent, laminar profile along the first ramp, with a gradual spanwise broadening of the flow as it approaches the front of the second ramp. At the second ramp, the gas is diverted along the inclination of the second ramp. As the gas continues along the second 
Runs: No Flow

$\mathrm{P}_{2}$ : 0.08 psi

Trip: 17-Hole

Target Rate: 0.0 SLPM

$\mathrm{P}_{\text {manifold }} / \mathrm{P}_{2}: 0$

$\mathrm{X}_{\text {trans }} / \mathrm{L}$ : - (Transition Location)

$\delta: 0.12$ inch (BL Thickness)
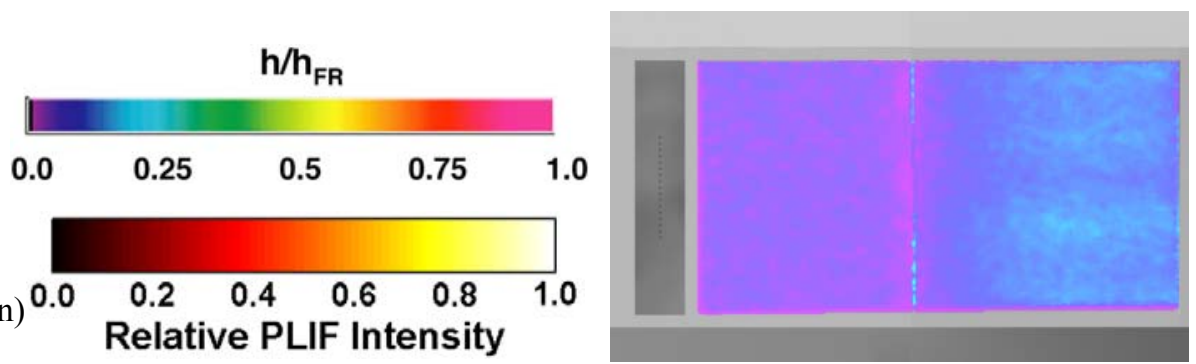

Runs: 6, 14

Trip: 17-Hole

Target Rate: $\sim 0.7$ SLPM

$\mathrm{P}_{\text {manifold }} / \mathrm{P}_{2}: 5$

$\mathrm{X}_{\text {trans }} / \mathrm{L}: 0.45$

Runs: 7, 13

Trip 17-Hole

Target Rate: $\sim 3.6$ SLPM

$\mathrm{P}_{\text {manifold }} / \mathrm{P}_{2}: 20$

$\mathrm{X}_{\text {trans }} / \mathrm{L}: 0.30$

Runs: 4,12

Trip: 17-Hole

Target Rate: $\sim 15.3$ SLPM

$\mathrm{P}_{\text {manifold }} / \mathrm{P}_{2}: 81.25$

$\mathrm{X}_{\text {trans }} / \mathrm{L}: 0.25$
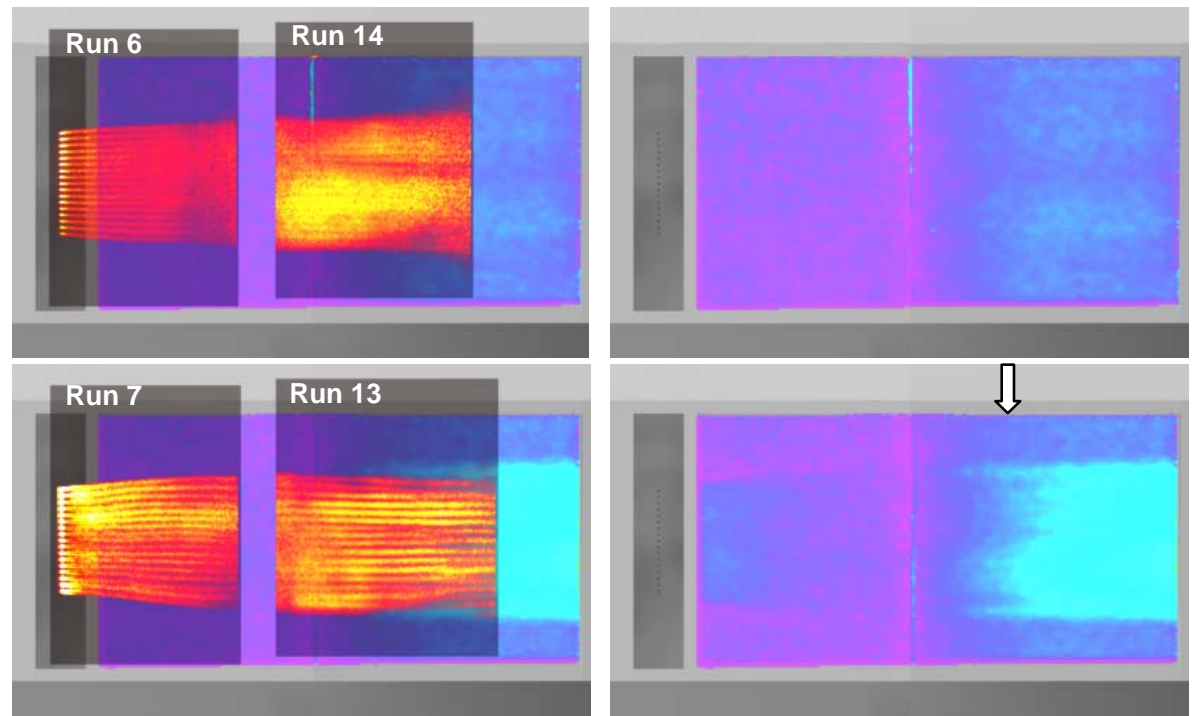

Runs: 5,15

Trip: 17-Hole

Target Rate: $~ 31.6$ SLPM

$\mathrm{P}_{\text {manifold }} / \mathrm{P}_{2}: 160$

$\mathrm{X}_{\text {trans }} / \mathrm{L}: 0.22$

Runs: 3, 16

Trip: 17-Hole

Target Rate: $\sim 51$ SLPM

$\mathrm{P}_{\text {manifold }} / \mathrm{P}_{2}: 248.75$

$\mathrm{X}_{\text {trans }} / \mathrm{L}: 0.22$
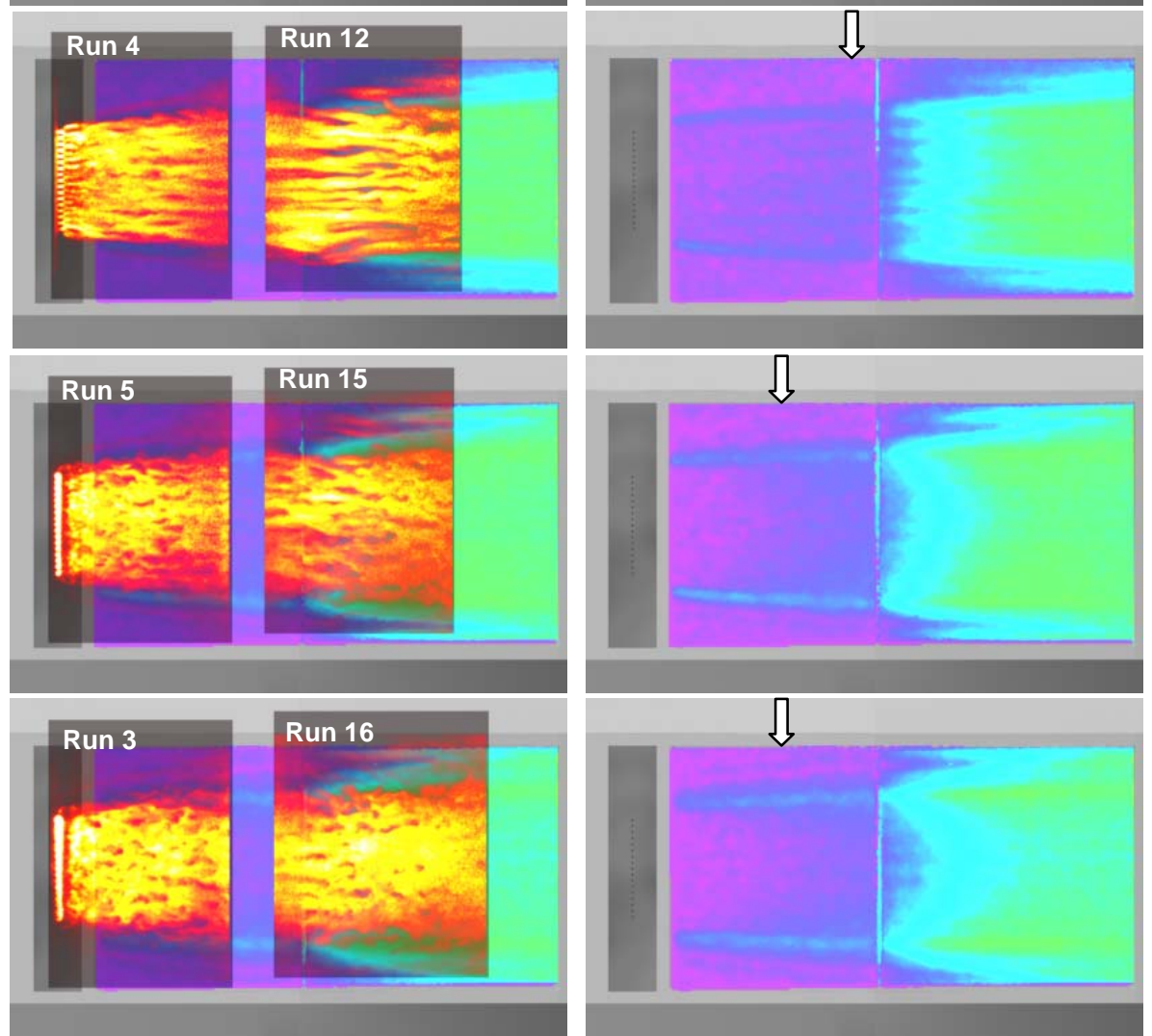

Figure 5. Comparison of phosphor-thermography surface heating profile with PLIF images taken at 0.075 inches off of the first and second compression ramps. Arrows signify transition point determined in Ref. 2.

American Institute of Aeronautics and Astronautics 


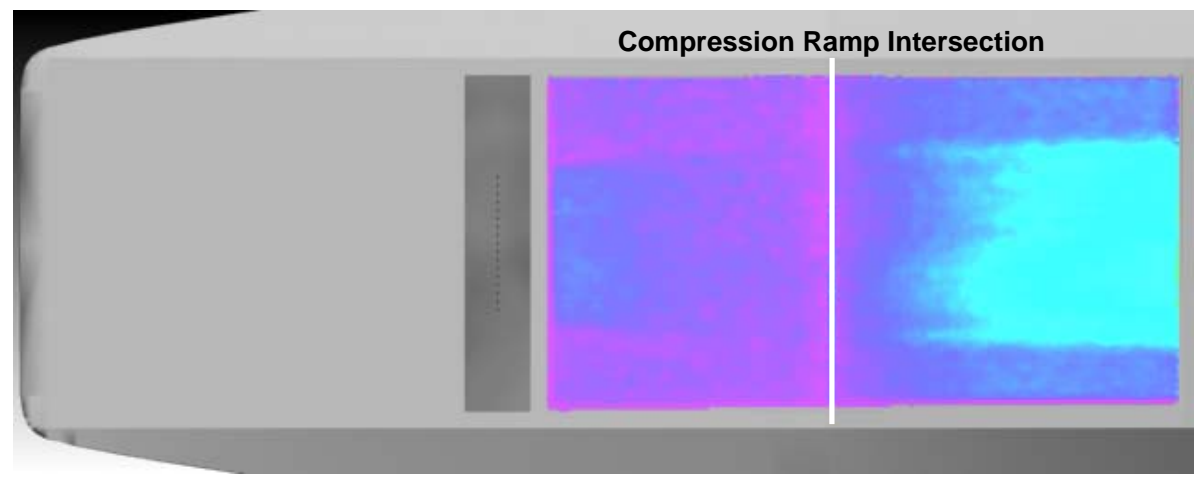

(a) Phosphor-thermography results with no overlaid PLIF images
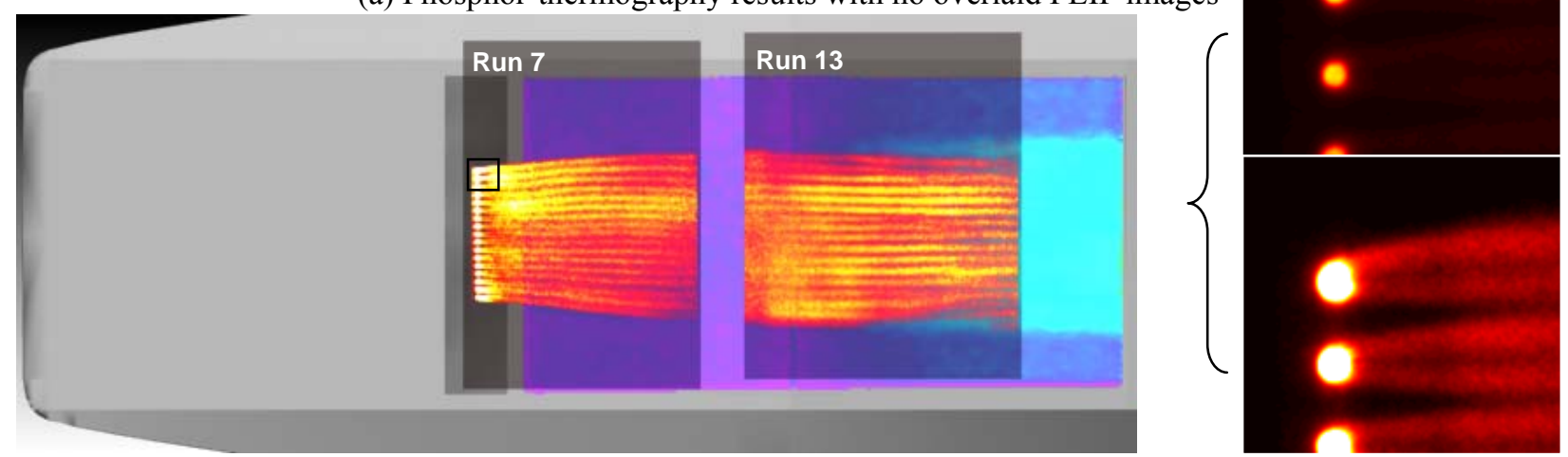

(b) PLIF imaging 0.075-inches off-body
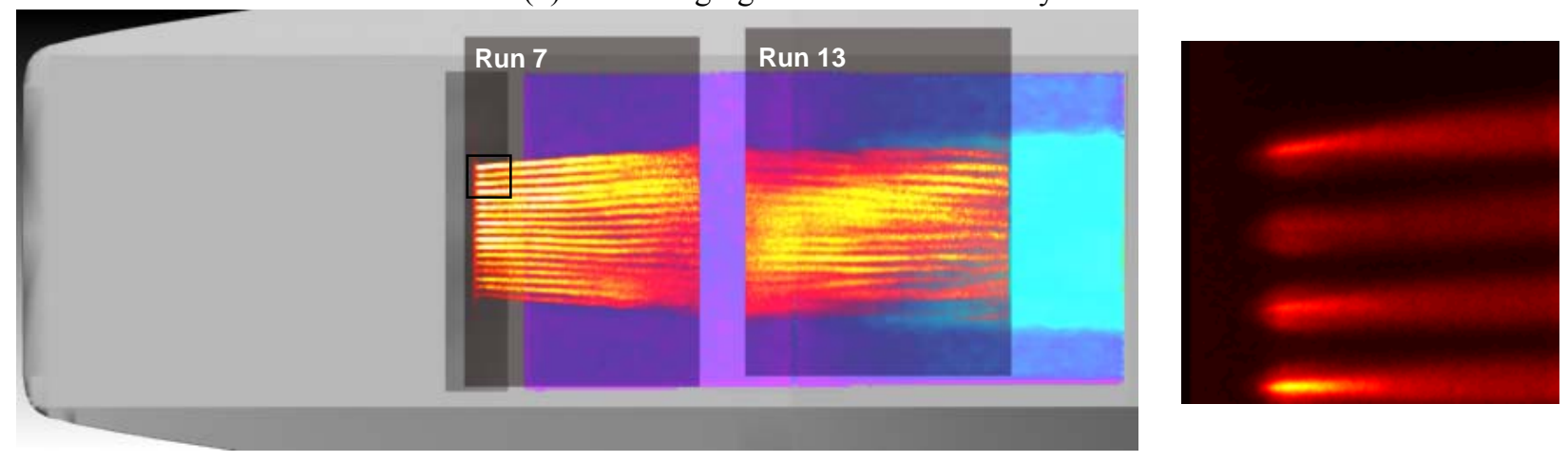

(c) PLIF imaging 0.1125-inches off-body
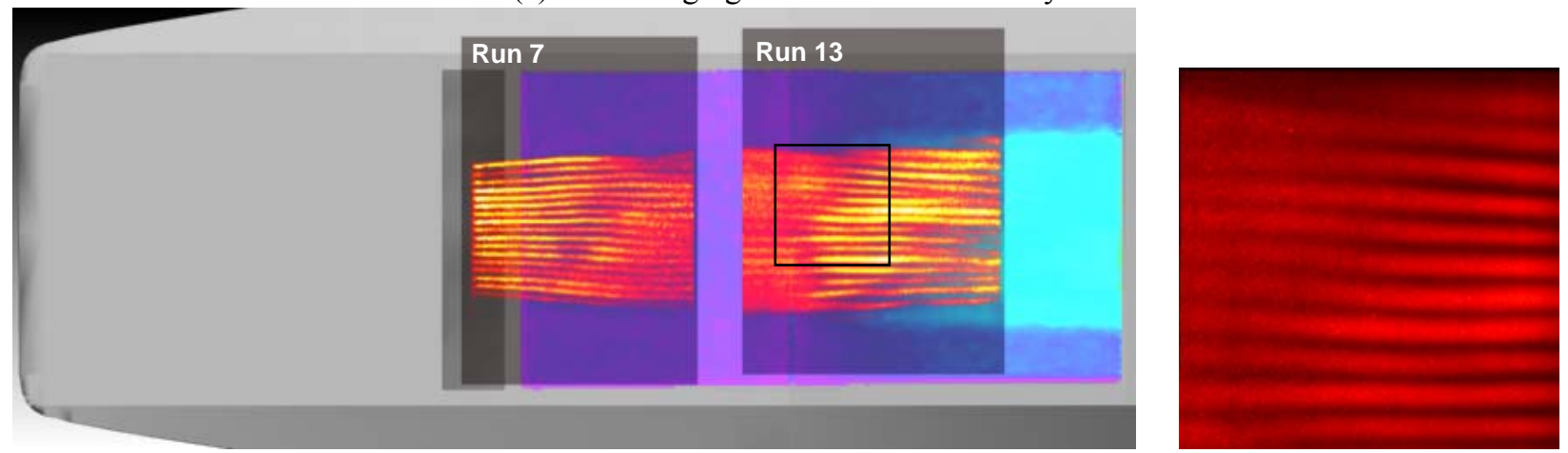

(d) PLIF imaging 0.15-inches off-body

Figure 6. Runs 7 and 13 with a flow rate of $\sim 3.6$ slpm (corresponding to Run 83 in Ref. 2). Higherresolution PLIF images (taken with a second camera) are shown on the right, with black boxes indicating their location relative to the images on the left. 


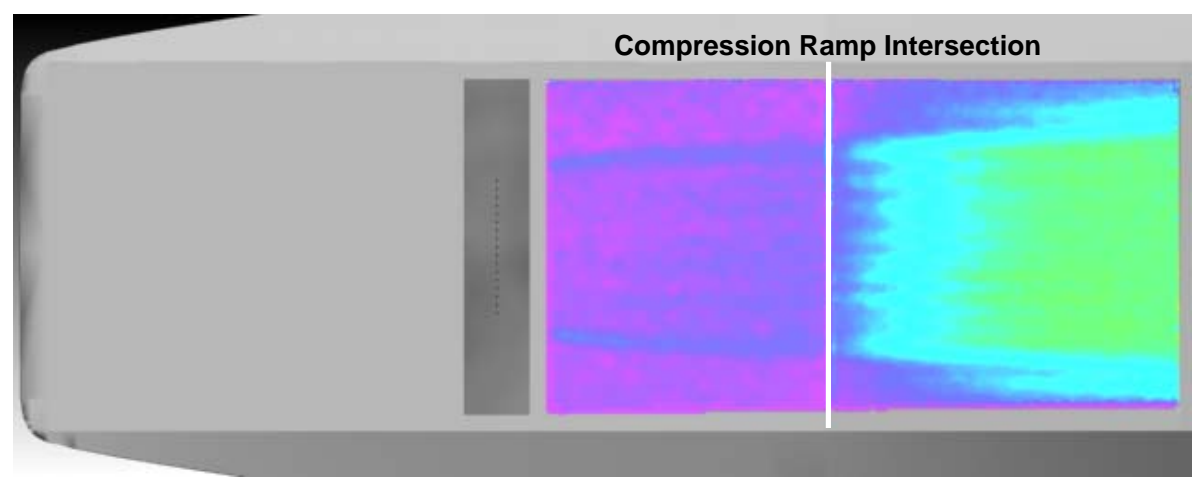

(a) Phosphor-thermography results with no overlaid PLIF images
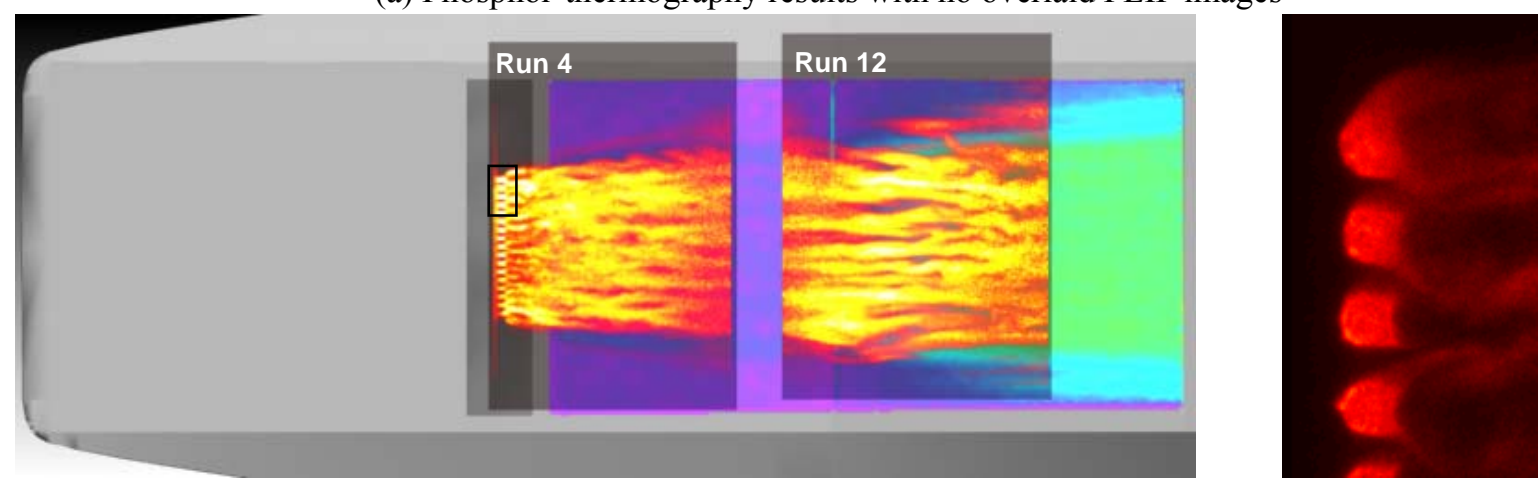

(b) PLIF imaging 0.075-inches off-body
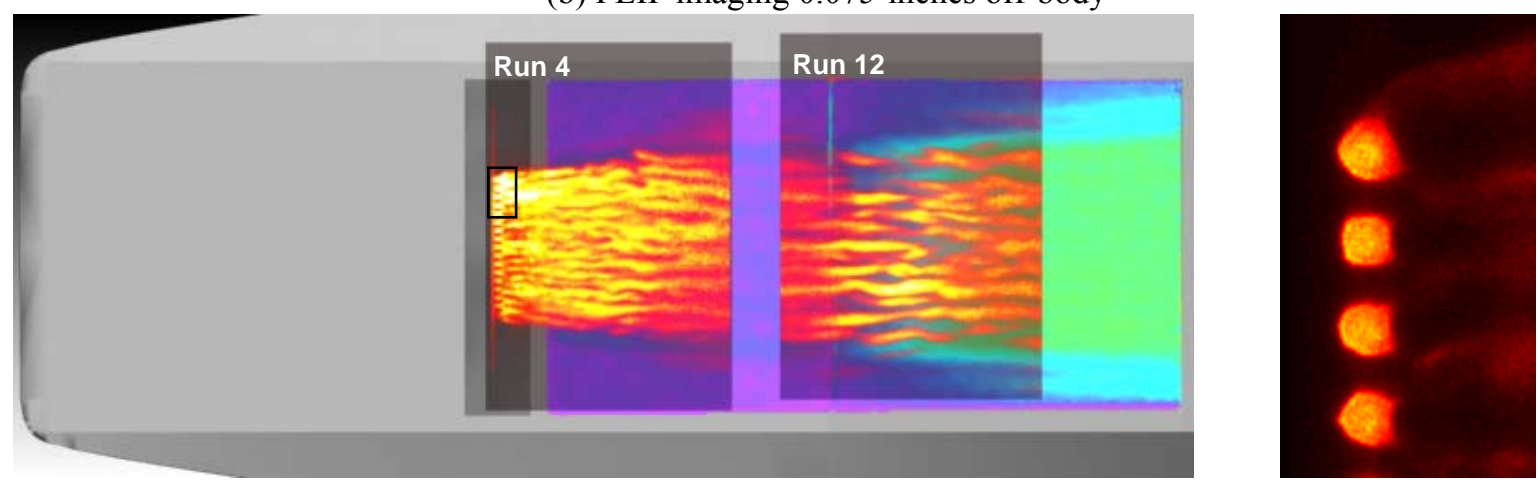

(c) PLIF imaging 0.1125-inches off-body
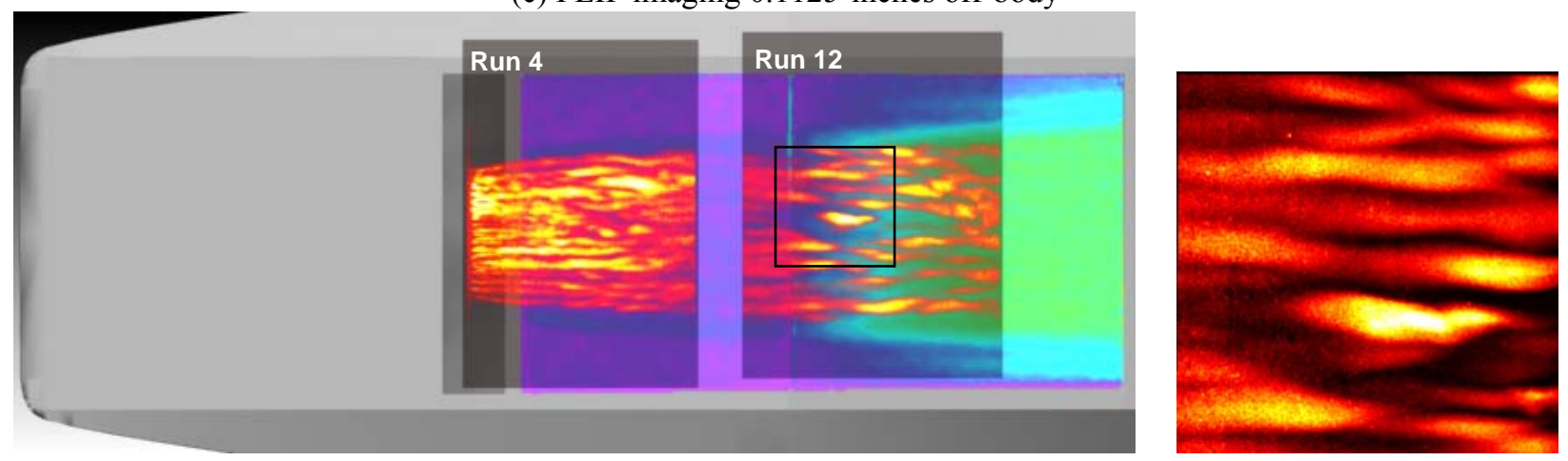

(d) PLIF imaging 0.15-inches off-body

Figure 7. Runs 4 and 12 with a flow rate of $\sim 15.3 \mathrm{slpm}$ (corresponding to Run 81 in Ref. 2. Higherresolution PLIF images (taken with a second camera) are shown on the right, with black boxes indicating their location relative to the images on the left.

American Institute of Aeronautics and Astronautics 092407 


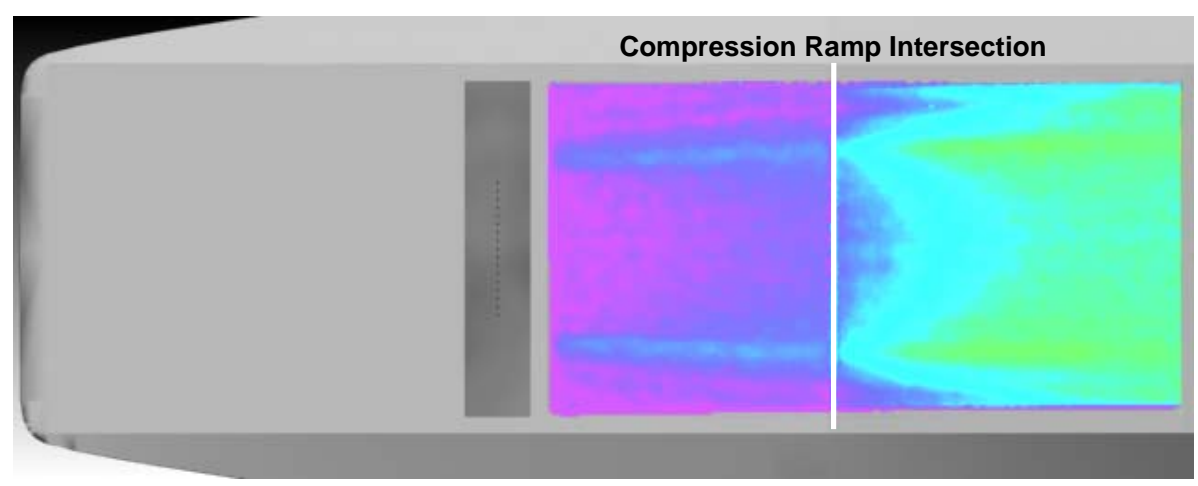

(a) Phosphor-thermography results with no overlaid PLIF images
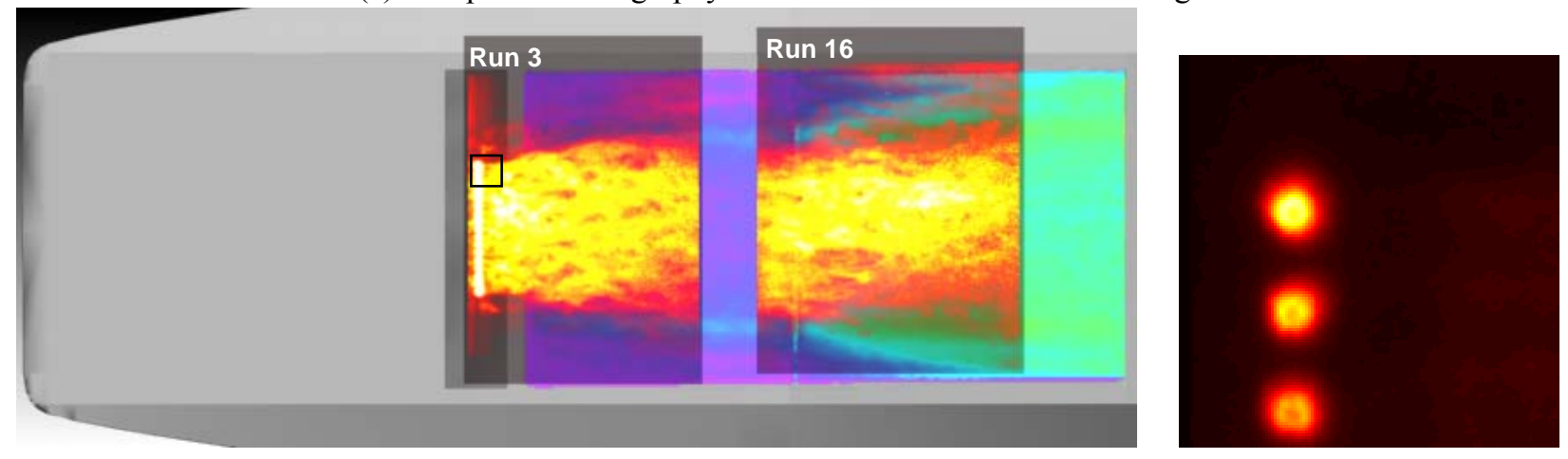

(b) PLIF imaging 0.05-inches off-body
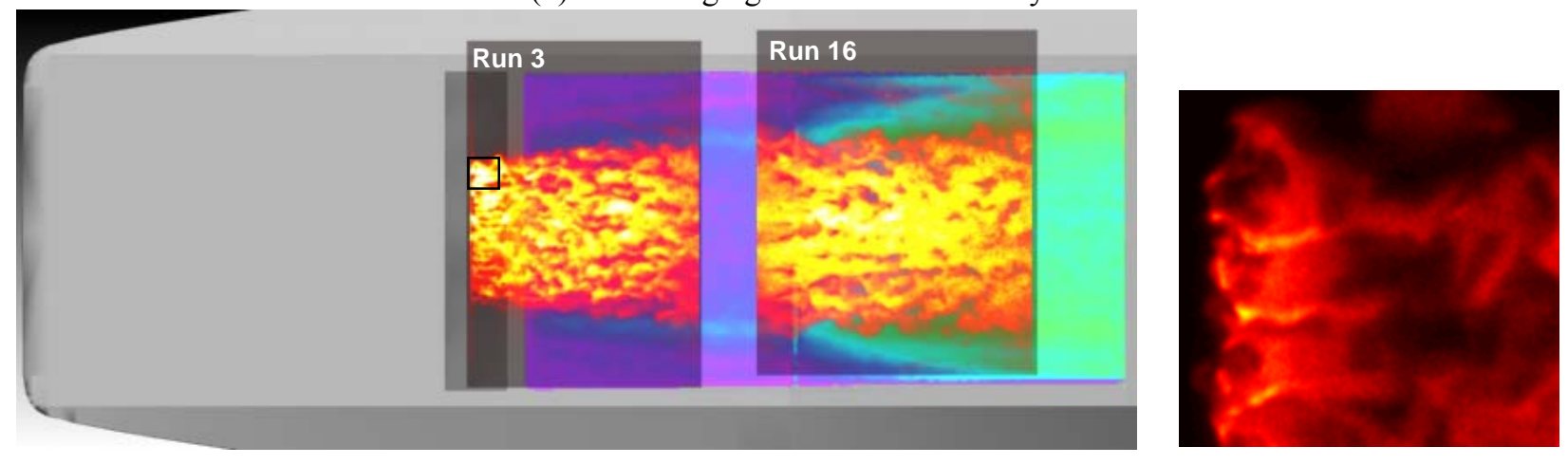

(c) PLIF imaging 0.15-inches off-body
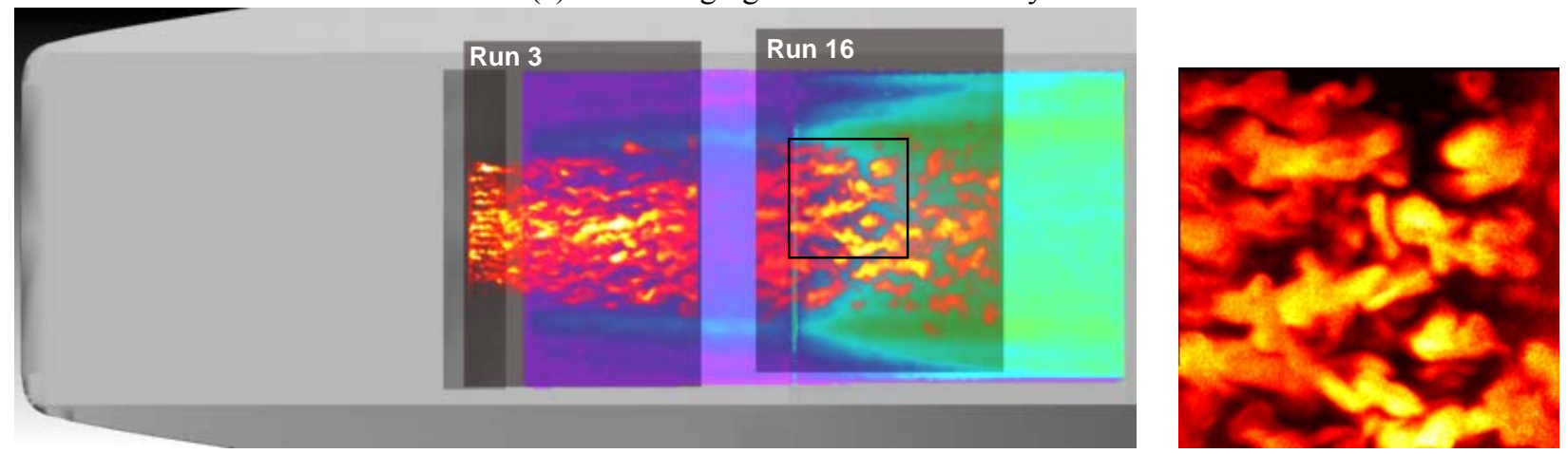

(d) PLIF imaging 0.25-inches off-body

Figure 8. Run 3 and 16 with a flow rate of $\sim 51$ slpm (corresponding to Run 79 in Ref. 2). Higher-resolution PLIF images (taken with a second camera) are shown on the right, with black boxes indicating their location relative to the images on the left. 


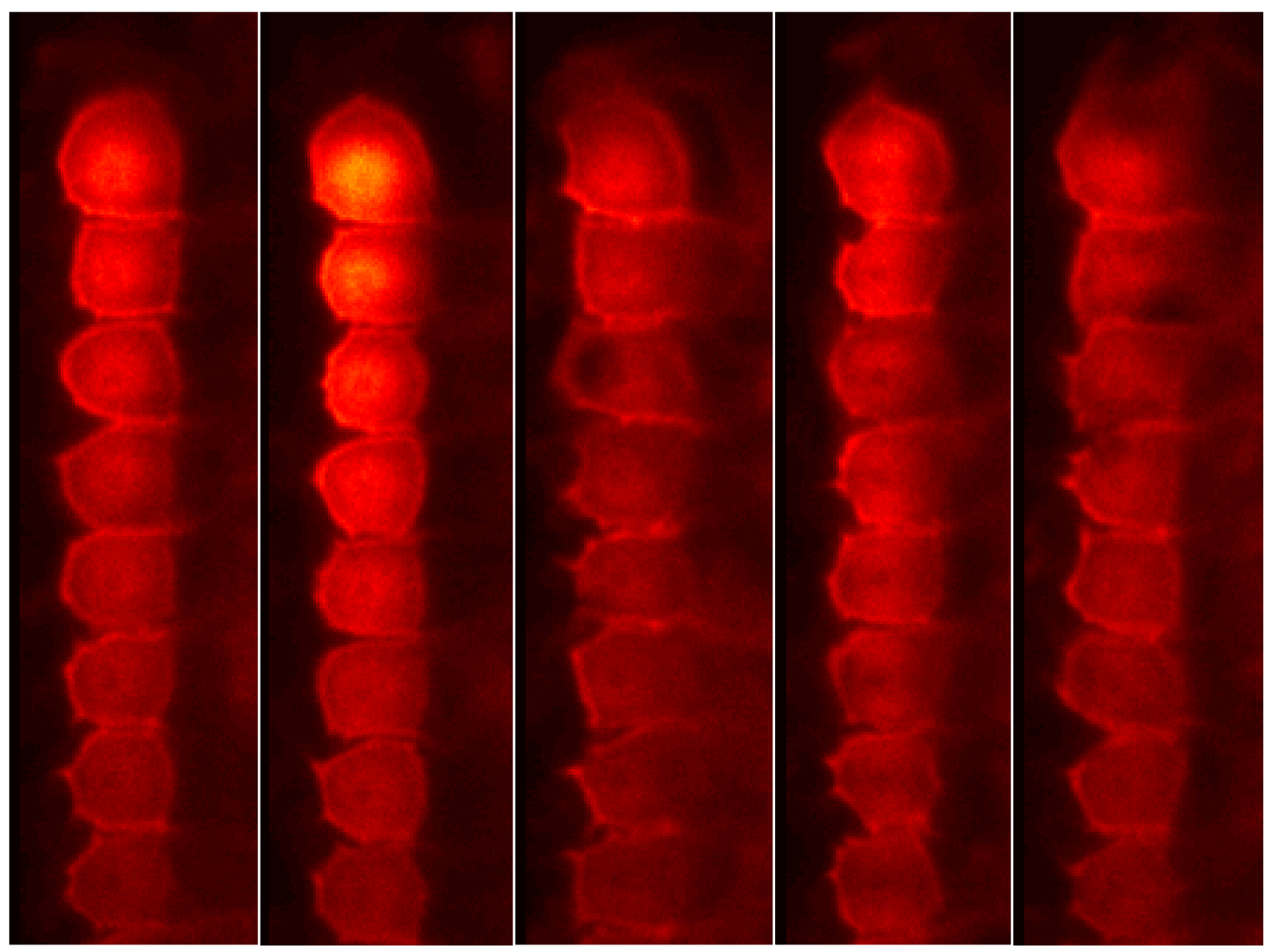

\section{Figure 9. Run 3 with a flow rate of $\sim 50$ slpm (corresponding to Run 79 in Ref. 2. PLIF images taken} sequentially between 0.103 -inches and 0.123 -inches at linearly spaced intervals.

ramp, the outer streaklines were generally observed to have a diminished fluorescence intensity relative to the inner ones and appear to wobble more than the center streaklines (Figure 6d, near field image).

Examination of the corresponding phosphor-thermography image shows that near the front edge of the first phosphor plate, the surface heating increases and has a profile matching the flow pattern (observed in the PLIF images) near the surface, immediately downstream of the trip insert. As the flow progresses downstream, the heat transferred to the vehicle body gradually decreases. After the primary flow crosses the intersection point of the two compression ramps, another increase in surface heating was observed. The two lines extending upstream along the upper and lower portion of the increased heating region suggest that the outer streaklines are interacting with a streamwise vortex originating from the flow separation caused by the fluid injection.

Increasing the blowing mass flow rate to approximately $15.3 \mathrm{slpm}$ produces the flow pattern shown in Figure 7. Near the model surface, the primary flow emanating from the trip holes (Figure $7 \mathrm{~b}$ ) appears to become unsteady immediately and remains that way as it proceeds downstream along the first ramp. Similarly, imaging of the flow further off-body (Figure 7c and d) shows the unsteady nature of the gas jets. As the gas passes over the second ramp and progresses downstream, this unsteadiness is amplified, and the individual streams of blown gas begin to separate from each other. This separation was more noticeable as the laser sheet scanned away from the model surface.

Thin, low intensity, uniform streams of gas were observed near the model surface along the first compression ramp (Figure $7 \mathrm{~b}$ and Figure $7 \mathrm{c}$ ) far outside of the primary flow. These streams of gas appeared to have originated from either the edges of the outer trip holes or in front of the trip holes. As these gas streams proceeded over the first plate, they diverged from the primary gas flow, resulting in the formation of a thin region between this secondary and the primary flow where nitric oxide fluorescence was not observed. After passing over the ramp intersection, the outer gas streams became increasingly unsteady while moving along the outer edges of the second plate. A gap was again observed between the outer and inner gas flows, and strong fluctuations of the inner flow were also observed. Close inspection of this gap along both the first and second compression ramps suggests that a vortex structure might be passing through this region near the surface.

Comparing these PLIF images with the corresponding phosphor-thermography image (Figure 7) reveals that the gap region along the first plate is correlated with an augmentation in surface heating relative to the region containing the inner and outer gas flows. It appears that this heating trend continues onto the second ramp, although heating 
directly below the primary flow region also increases (light blue coloration) very near the intersection of the two ramps. As the primary flow continues along the second ramp, heating continues to increase (green coloration).

Figure 8 shows the resulting boundary layer flow with the highest target blowing flow rate, $49.7 \mathrm{slpm}$, and the corresponding phosphor-thermography image. Near the surface of the first and second compression ramps, the observed gas flow structures appeared highly mixed and turbulent immediately after the fluid injection (Figure 8b). The flow continued to broaden along the length of both compression ramps. As in the $15 \mathrm{slpm}$ blowing flowrate case, a secondary outer flow again appears to have originated from either the outer trip holes or in front of the trip holes. Unlike the previous case, however, no gap is observed. Rather, the region between this outer gas stream and the inner, primary flow presumably contains nitric oxide gas entrained by a vortex structure, which continues along the second ramp. Evidence for the existence of this vortex is seen by the diagonal structures in this outside flow. The region containing this vortex is significantly wider than the region observed in Figure 7, a fact which is consistent with the wider heating augmentation footprint seen in the phosphor-thermography image. Figure $8 \mathrm{~b}$ shows that this possible vortex flow is more pronounced along the second ramp. As the secondary (outer) flow continues to move downstream, it appears to move further away from the primary inner flow (with a corresponding growth of the outer vortices) while becoming increasingly unsteady, as evidenced by the alternating bands of fluorescence. As this outer gas stream passes over the second ramp, it partially merges with the vortex (Figure $8 \mathrm{~b}$ and c). Far from the vehicle surface, the unsteady structures become increasingly distinct as the flow proceeds downstream.

Figure 9 shows a sequence of images taken while scanning the laser sheet away from the model surface. The spike structures shown became visible a small distance away from jet exit (at approximately 0.58 inches) and remained visible until approximately 0.183 inches off of the model surface. Beyond this point, only the broken turbulent structure shown in Figure 8d (along the first compression ramp) was observed. These spike structures have been identified as sources of instability, promoting transition to turbulence and under expanded chance. ${ }^{17}$

The phosphor-thermography image in Figure 8a shows that the region containing the vortex is significantly broader as compared to the $15.3 \mathrm{slpm}$ flow rate case. The phosphor image also shows that along the path of the outer gas stream on the first compression ramp, the surface heating is reduced. However, a slight increase in surface heating is observed at the outer boundary of this gas stream, which also corresponds to the outer edge of the gas stream that was visibly unsteady near the front edge of the second plate. Figure $8 \mathrm{a}$ and Figure $8 \mathrm{~b}$ seem to confirm this assertion, as the increased unsteadiness observed in the PLIF images correspond to increased heating spikes located near the outer edges of the second phosphor plate. In the region encompassing the primary flow structures, a bowing of the surface heating profile is observed. Analysis of PLIF images taken near the surface (Figure $8 \mathrm{~b}$ ) - and even those closer to the surface (not shown) - does not appear to provide any insight as to the cause of this profile.

\section{B. Single-Hole Trip Configuration}

Figure 10 shows results for a single blowing jet using a plenum pressure of $3.78 \mathrm{psi}$, and a flow rate of $\sim 0.11 \mathrm{slpm}$. As the jet travels along the first compression ramp, the gas nearest the surface has a slightly broader profile than gas located further off of the surface. This can be attributed to the separation region flow passing around the jet and proceeding downstream along with the primary flow. Images taken away from the model surface (Figure $10 \mathrm{c}, \mathrm{d})$ show that the gas jet narrows and remains laminar. As this flow passes onto the second compression ramp, the gas nearest the surface (Figure 10b) is further broadened, yet remains laminar as it passes downstream. Figure 13 shows a planar image of the gas just slightly off of the model surface. This image highlights the broadening of the secondary gas flow as it passes onto the second compression ramp, which is also shown in Figure 13 where the laser sheet is nearly flush with the model surface. The noise observed in the image is a result of laser scattering off of the model surface. Planar images of the gas jet away from the surface show that gas jet maintains a similar profile to that seen passing over the first plate.

The phosphor-thermography image superimposed on the model in Figure 10 was acquired during a run with a slightly lower plenum pressure of $3.2 \mathrm{psi}$, a lower stagnation pressure of $1350 \mathrm{psi}$, and a 3-hole blowing jet configuration that consisted of a 0.01 inch diameter, 17-hole trip insert with a backing plate sandwiched between the plenum and trip mount. Figure 10a shows the 3-hole configuration similar to that used in the phosphorthermography test performed by Berry et al. ${ }^{2}$ (but not previously published) with the holes slightly enlarged for ease of viewing. The backing plate only allowed gas to pass through the center and outermost holes, so it has been assumed that interaction between jets is negligible along the first and second compression ramps. Along the first plate, nearly uniform surface heating was observed, with a narrow, reduced heating region immediately prior to the ramp intersection point. Only a slight increase in surface heating is visible along each gas path (faint blue lines). On the second ramp, on either side of the primary gas flow, a significant increase in surface heating was observed. This is highlighted by the two lines of increased surface heating observed on either side of the primary gas jet. While the 
phosphor images show some evidence of a pair of vortices (perhaps a horseshoe vortex) generating the streak-like heating pattern on the second plate, the PLIF images only show evidence of two vortices very near the point of injection. This is apparent in the near-field images, where there is a decrease in PLIF intensity directly downstream of the injection port. This apparent difference in flow behavior could be due to the two freestream unit Reynolds numbers applied in Ref. 2 and in this work, 1.85 million per foot and 1.96 million per foot, respectively.

As the flow rate was increased to $0.61 \mathrm{slpm}$ (approximate) with a plenum pressure of $17.4 \mathrm{psi}$, additional flow features are observed. Figure 11 shows the result of this case with a superimposed phosphor thermography image representing a slightly lower plenum pressure of 12.8 psi. In this case, the primary gas flow originating from the blowing jet remains laminar along the length of the first compression ramp until it approaches the ramp intersection point. As this gas stream approaches this point and passes onto the second ramp, it appears to separate into two gas streams which continue to interact and merge with one another as they proceed downstream (Figure 11b). Imaging further off of the model surface (Figure 11c) shows that the primary gas stream becomes unsteady along the second ramp, forming a single vortex structure. Imaging farther away from the surface shows that the primary gas stream becomes unsteady as it passes onto the second compression ramp (Figure 11d). The secondary gas flow originating from the separation region near the injection passes around the primary jet and travels downstream with the primary gas jet. As this secondary flow approaches the second compression ramp, it begins to diverge away from the primary gas stream. As the secondary flow passes onto the second ramp, it continues to separate from the primary flow, creating a gap similar to that observed in the 17-hole configuration tests.

Figure 11 again shows a phosphor-thermography image obtained during the test by Berry et al. ${ }^{2}$ using the 3-hole blowing trip configuration. Observation of the phosphphor-thermography image in Figure 11 shows that heating beneath the primary and secondary gas flows is fairly uniform until approximately $3 / 4$ of the way down the first plate. This corresponds to point where the secondary gas stream begins to separate from the primary flow. As the secondary flow continues onto the second ramp, it passes along the outer edge of the increased surface heating region. This increased heating region, which appears as two lateral blue lines in Figure 11a, corresponds to the gap region formed between the primary and secondary gas streams and containing possible vortices which are not visible due to an absence of seeded $\mathrm{NO}$ at their point of origin.

Figure 12 represents a blowing rate of $1.8 \mathrm{slpm}$ and a plenum pressure exceeding the measurable range of the plenum piezoresistive pressure sensor. No phosphor-thermography images were available corresponding to this flow condition. Flow structures seen in the PLIF images are similar to those observed in the $0.61 \mathrm{slpm}$ case (Figure 11). The near surface image shows that along the first compression ramp, the secondary flow divergence from the primary gas stream occurs almost immediately after passing around the blowing gas jet. As the secondary gas passes onto the second compression ramp, it significantly broadens and becomes unsteady. Farther off-body, this secondary gas stream had a similar profile along the first plate and became unsteady as it passed over the second plate without any observable broadening. As this gas stream proceeded along the first ramp, it remained laminar but displayed a small level of unsteadiness approximately halfway down the plate. As this gas passed over the ramp intersection point, the near surface images showed that the magnitude of its unsteadiness continued to increase, apparently affected by the vortices present in the gap between the primary and secondary flow. Images taken farther off of the surface indicate that primary gas stream is strongly unsteady and appears to form another vortex which begins to breaks down approximately halfway down the second ramp. Compared to the lower flow rate cases this vortex appears to have a higher rate of rotation at least spatially.

\section{Discussion}

For the 17-hole blowing trip configuration, the rate of the mass flow used to disturb the boundary layer had a significant affect on the magnitude and distribution of surface heating. As the flow rate was increased, the crosssectional area of the jets appeared to increasingly restrict the amount of separation region gas allowed to pass between individual jets. This relation between flow restriction and flow rate may be proportional to the separation region size and the residence time of the gas trapped in front of the jets. A schematic showing boundary layer tripping methods using both solid spheres and blowing jets is provided in Figure 14a. Figure 14a also shows the separation region existing ahead of the disturbance origin. As the flow rate was increased, the trapped gas in front of the jets possibly acquired an increased amount of heat energy transferred from oncoming air stream. This phenomenon is more visible in a stereoscopic analysis of the flow reported by Danehy et al. ${ }^{8}$. This increased amount of heat appeared to then be transferred to the surface via a horseshoe-like vortex originating from the separation region, which passed around the end trip holes, and distributed this heat energy along the first and second compression ramps. This transfer mechanism appears be confirmed by the surface heating streaks observed 


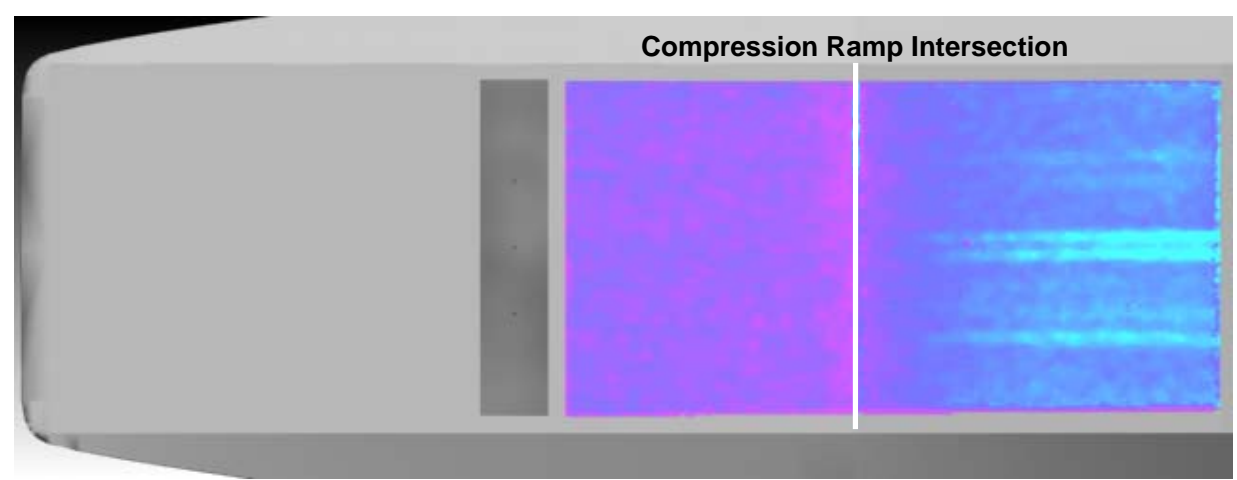

(a) Phosphor-thermography results with no overlaid PLIF images at manifold pressure of $3.2 \mathrm{psi}$
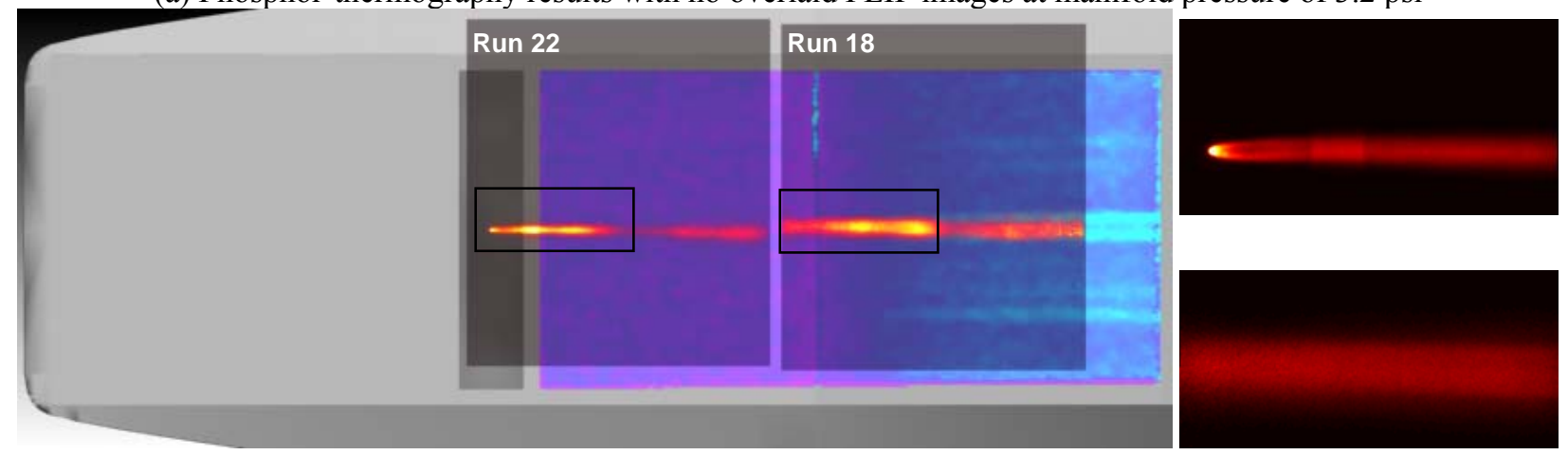

(b) PLIF imaging 0.05-inches off-body
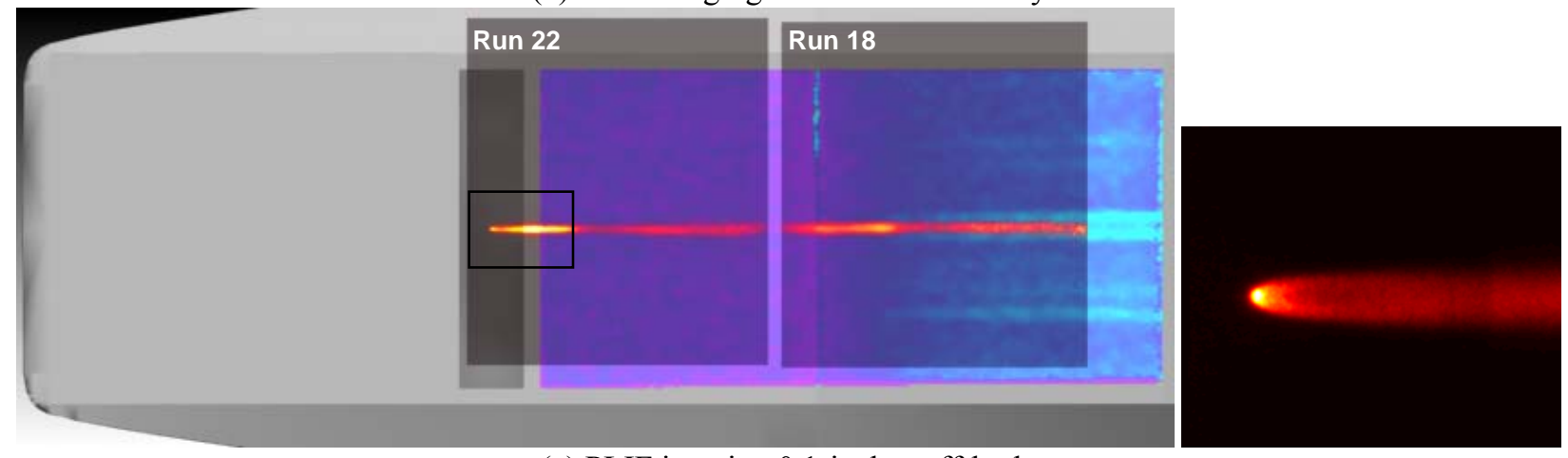

(c) PLIF imaging 0.1-inches off-body

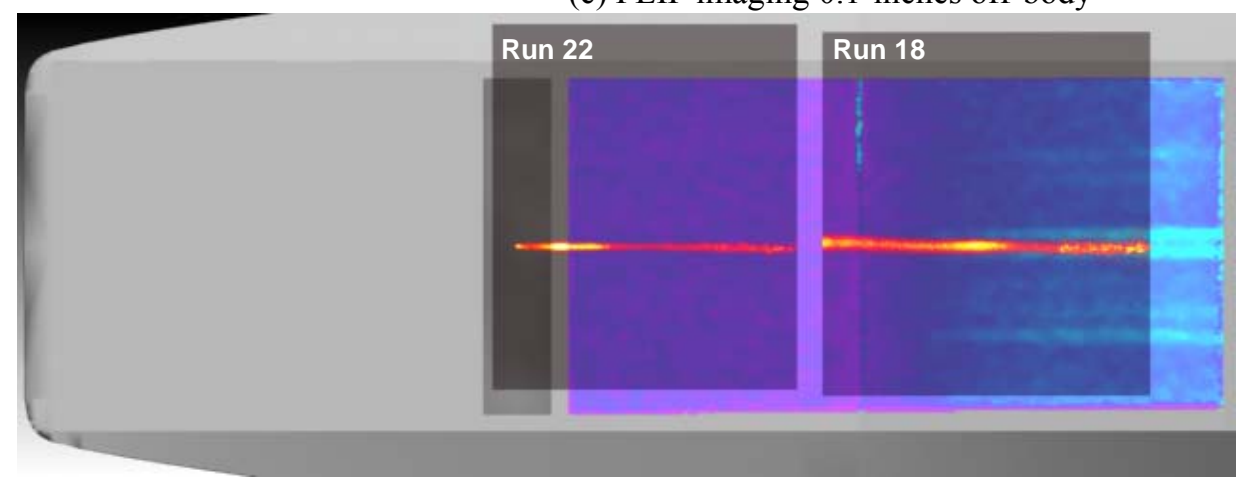

(d) PLIF imaging 0.15-inches off-body

Figure 10. Runs 22 and 18 with a flow rate of $\sim 0.11 \mathrm{slpm}$ (corresponding to Run 30 in Ref. 2). For the PLIF images, the manifold pressure was approximately 3.79 psia. Higher-resolution PLIF images (taken with a second camera) are shown on the right, with black boxes indicating their location relative to the images on the left. 


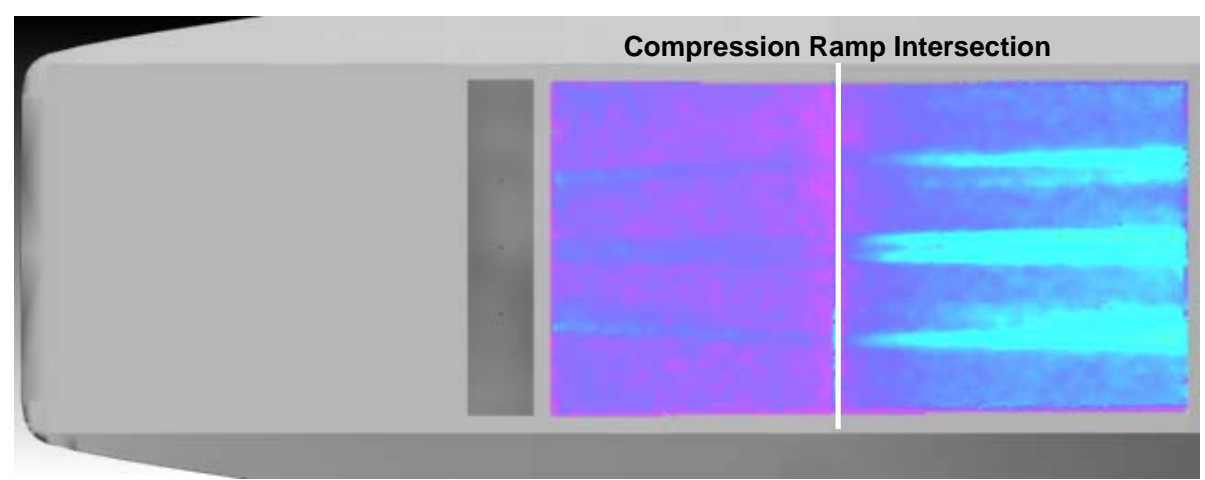

(a) Phosphor-thermography results with no overlaid PLIF images at manifold pressure of $12.8 \mathrm{psi}$
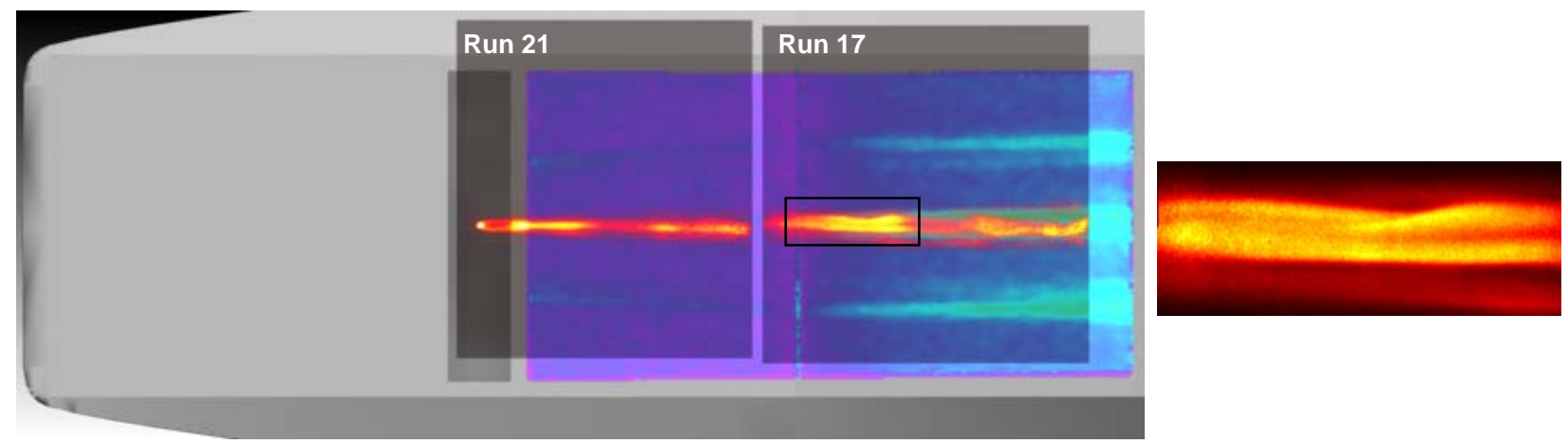

(b) PLIF imaging 0.05-inches off-body
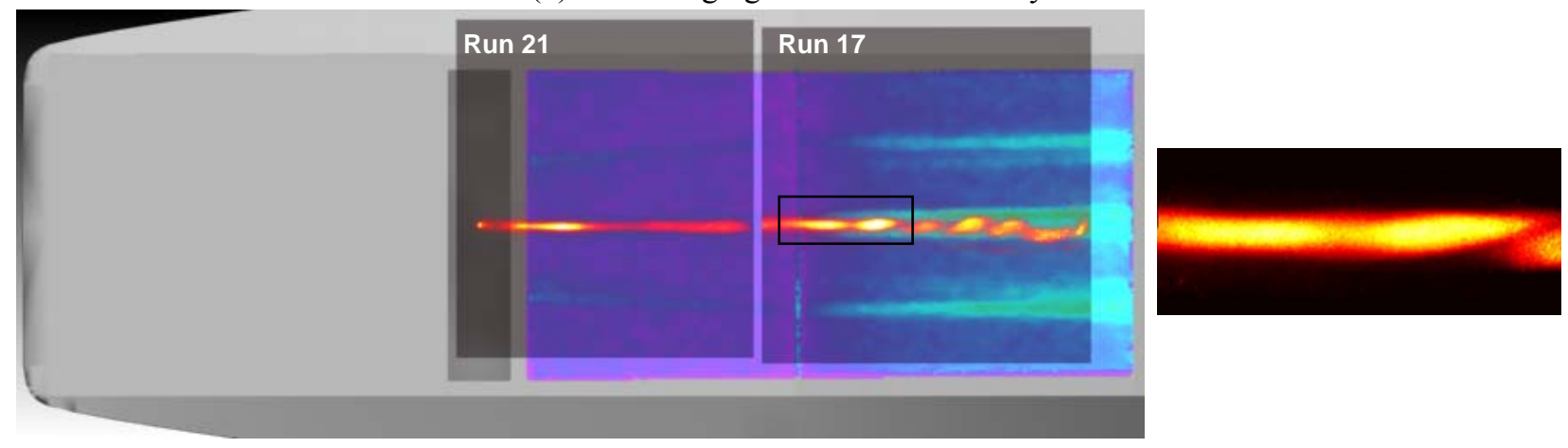

(c) PLIF imaging 0.15-inches off-body

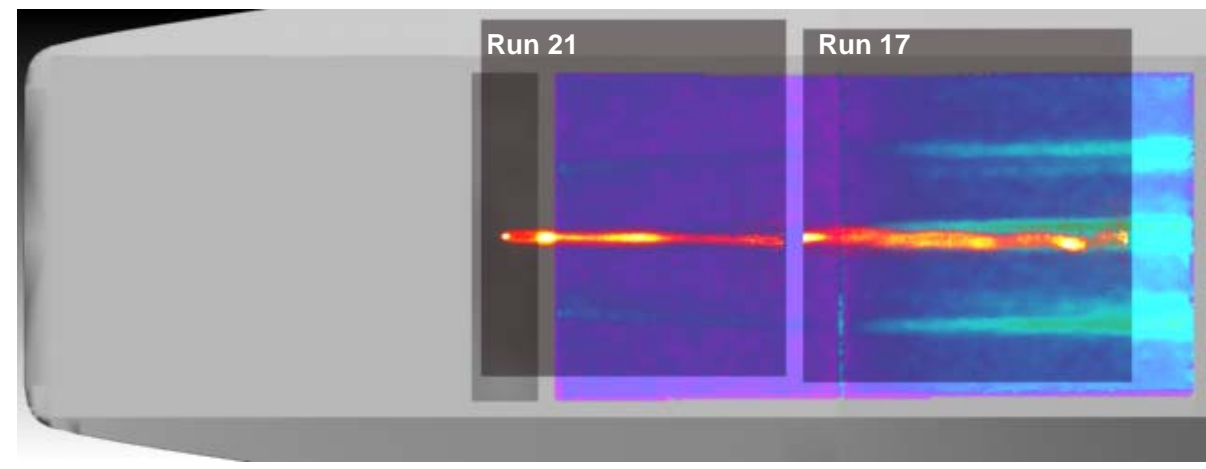

(d) PLIF imaging 0.25-inches off-body

Figure 11. Runs 21 and 17 with a flow rate of $\sim 0.61 \mathrm{slpm}$ (corresponding to Run 32 in Ref. 2. For the PLIF images, the manifold pressure was approximately 17.4 psia. Higher-resolution PLIF images (taken with a second camera) are shown on the right, with black boxes indicating their location relative to the images on the left. 


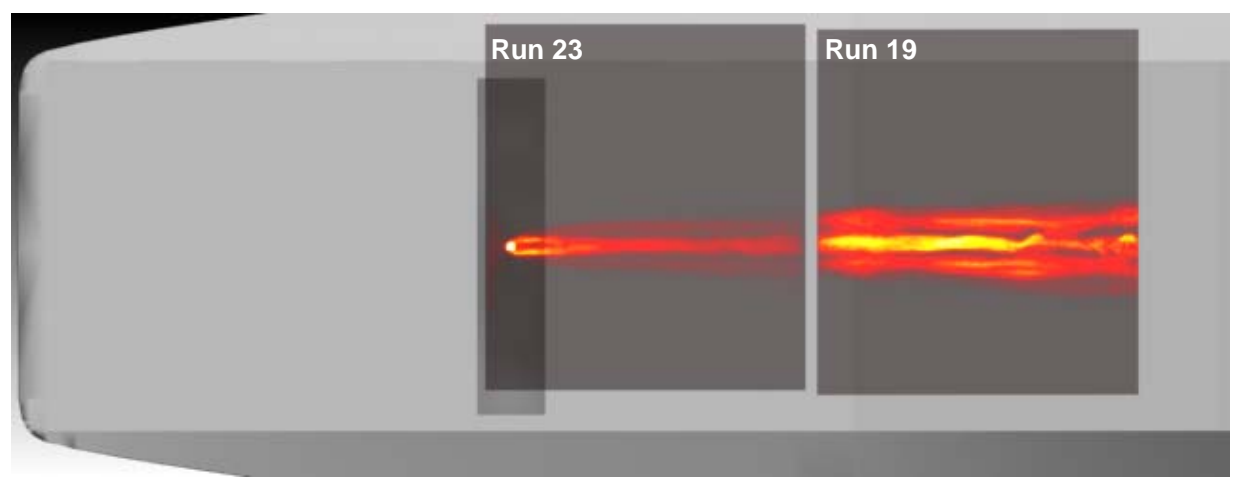

(a) PLIF imaging 0.05-inches off-body

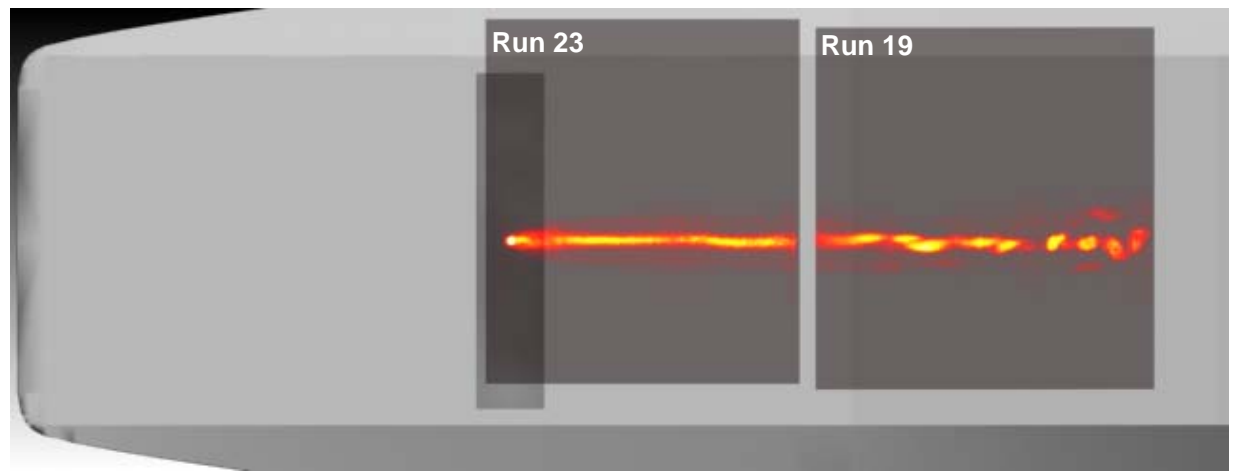

(b) PLIF imaging 0.2-inches off-body

Figure 12. Runs 23 and 19 with a flow rate of $\sim 1.8 \mathrm{slpm}$. In this run, the manifold pressure exceeded the range of the sensor, and was therefore not known.

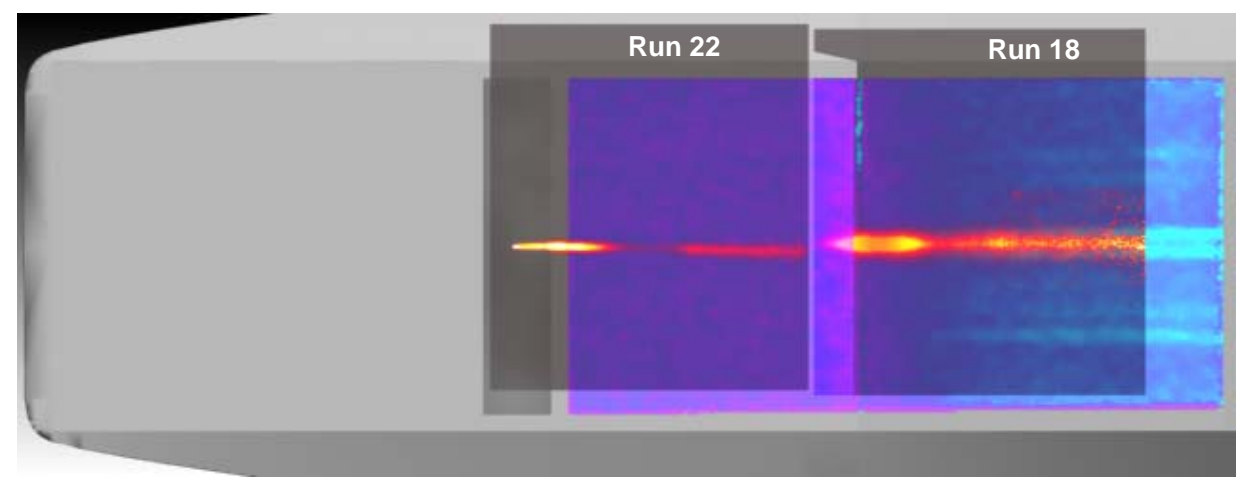

Figure 13. Flow rate of $\sim 0.11$ slpm with manifold pressure of approximately 3.79 psia. These images were taken with the laser sheet was very near surface, resulting in some noise (noticeable in the PLIF image on the right). Note the broadening of the laminar jet as it comes into contact with the second compression ramp. Corresponding phosphor-thermography image was for a manifold pressure of 3.2 psia. 
in the phosphor-thermography images in Figure 6 and Figure 7. At the highest flow rate $(51 \mathrm{slpm})$, it appeared that the vortex originating in the separation region entrained a small quantity of $\mathrm{NO}$ gas emanating from the jet, which became visible between the primary and secondary gas streams. For the lower flow rates, gas trapped in the separation region easily passes around the individual jets and distributes any heat transferred to it in that region to the surface as it proceeds downstream. This phenomenon is apparent in Figure 15 where each of these cases are presented with an increased contrast level that, while saturating the signal of the jets, shows trace amounts of NO out ahead of the jet in the $51 \mathrm{slpm}$ flow case but not the $3 \mathrm{slpm}$ flow case. This argument may also explain the apparent decrease in heating downstream of injection on the first plate for the higher flow rate cases: cool injected gas jets have formed a fence to prevent the hot stagnant gases from reaching the plate. Furthermore, some of the cool injected gases are flowing over the plate, possibly reducing the heat transfer.

The point of transition $\left(\mathrm{X}_{\text {trans }} / \mathrm{L}\right)$ determined through analysis of phosphor-thermography surface heating profiles differs from the flow transition point visualized through application PLIF. For the lower flowrate cases (i.e. 0.7 slpm and $3.6 \mathrm{slpm}$ cases), the primary flow observed with PLIF appears to remain laminar along the second compression ramp (with the exception of the outermost streaklines). For the higher flowrate cases (i.e. $15.3 \mathrm{slpm}$ and greater), the PLIF images show that the blowing jets cause transition almost immediately after exiting the trip orifices. The apparent difference observed between the PLIF images and phosphor-thermography measurements is
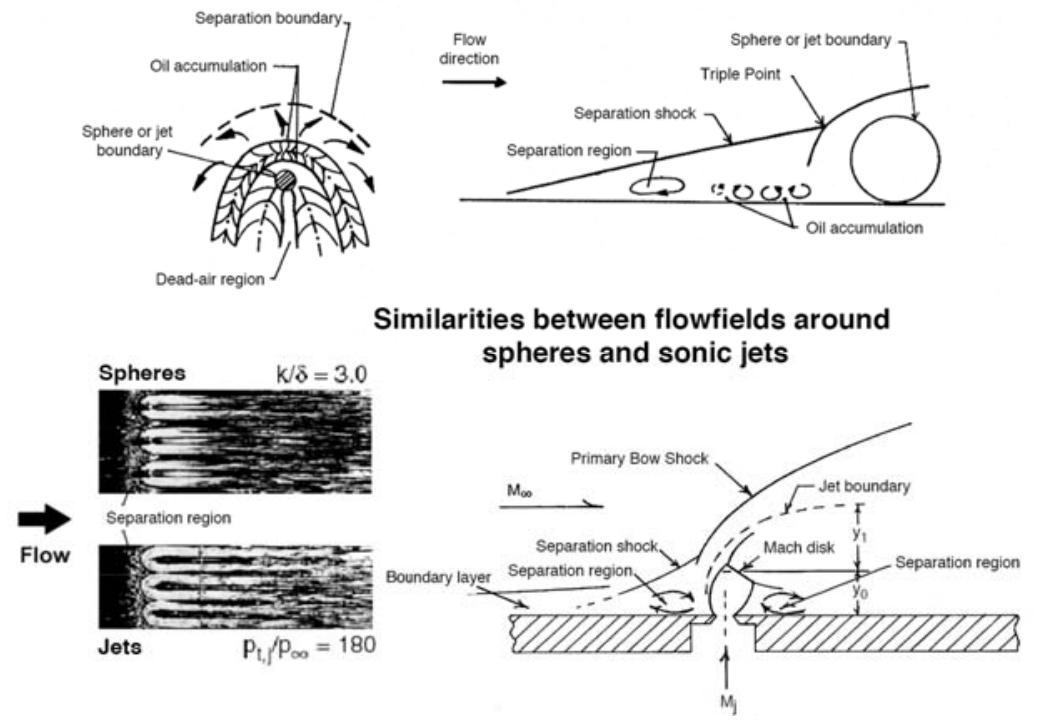

(a)

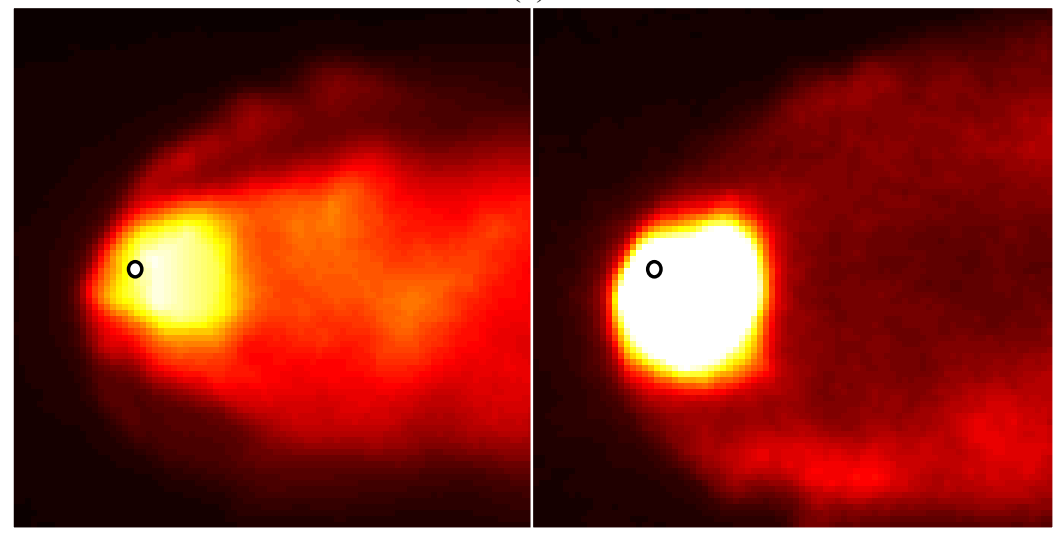

(b)

Figure 14. Description of sonic jets adapted from Refs. 7 and 2 (upper schematic). PLIF visualization of jet flow approximately 0.003 inches off-body (lower-left PLIF image) and at 0.062-inches off-body (lower-right PLIF image). Dot shows the approximate jet size and position. not easily explained When viewing PLIF images very near the surface (within the thickness of the laser sheet), the flow appears to have a more constant, uniform fluorescence profile. This might suggest several things: (1) that heating caused by transition could be occurring at much smaller scales near the surface than what can currently be resolved in the current experiment, (2) that the heating is a result of flow events occurring throughout the boundary layer, and/or (3) some other flow structure, not observed with NO fluorescence, generates the increased heating.

In the case of the 3 slpm blowing rate, gas in the separation region immediately in front of the blowing jets appears to be heated by energy transferred to it by oncoming air, which is consequently altered and slowed by the presence of such jets. Due to the lower flow rate, the separation region shown in Figure 14a is relatively small. Consequently, gas in this region may easily passes between the jets and progresses downstream, transferring to the model surface the heat energy acquired while in the separation region.

As the flow rate was increased, the cross section of the blowing jet normal to the flow direction also increased. This most likely reduced the ability of the gas circulating within the separation region to pass between individual jets, resulting in an increased residence time and consequently more heat energy 
transferred to the gas in that region by the oncoming flow. Eventually the gas in this region-limited to traveling around this effective fence/flow blockage - passed along the outside of the jet front. This result seems to be supported by both the phosphor-thermography images and PLIF image taken near the surface where the 3 slpm flow case appeared to allow passage of gas through the blowing jets and the heat acquired by this bypass gas is dispersed to the surface near the front of the first phosphor plate. At the next highest flow rate considered $(15.3 \mathrm{slpm})$ the majority of the gas trapped in the separation region passes around the outer blowing jets, forming a vortex, and distributing heat as it proceeds downstream. A relatively small amount of gas passes between the jets and distributes heat downstream of the trips.

Another interesting flow feature observed were spike structures, which were observed for the highest applied flow rates. These structures, shown in Figure 9, appear to greatly contribute to the highly turbulent flow profiles observed at locations further off-body of the model. Figure $8 \mathrm{c}$ and $\mathrm{d}$ shows the turbulent nature of the flow, which occurs at or beyond the point where the spiked structures are first visible. Flow nearer to the surface, such as that visualized in Figure 8a, had no visible spikes and the downstream gas appeared less broken. A more detailed discussion of these structures is presented by Danehy et al. ${ }^{8}$, where stereoscopic imaging captured the propagation of the blown gas from the jet exit and into the boundary layer.

The single-hole blowing trip tests also demonstrate how the flow rate determines and affects the size of the separation region, gas stream profile, subsequent secondary and tertiary flow structure formation, and surface heating. Additionally, consideration of a smaller diameter ( 0.01 inch versus $0.02 \mathrm{inch})$, single-hole trip demonstrates that by applying a higher flow rate, the width of the surface region affected by the trip also increases. Examination of Figure 11a tends to confirm the assertion that the gas within the separation region is trapped ahead of the trips and is restricted from passing around the jets based upon the width of the surface heating profile directly behind the jet.

At increased blowing rates, while the primary flow along the first compression ramp remained consistently laminar in nature, the flow along the second compression ramp became increasingly unsteady. Figure 16 shows the existence of a corkscrew-like structure in a sequence of PLIF images imaged along the second compression ramp. The flow in this instance was disturbed with a $0.61 \mathrm{slpm}$ single jet. The structures were approximately 0.15 -inches above the model surface. As flow is increased further, the nature of the primary flow along the second compression ramp becomes increasingly unsteady, as is shown in Figure 12d.

At the highest applied flow rate of 1.9 slpm (Run 23 and 19), PLIF visualization very near the model surface (lower-left images in Figure 14b) shows gas passing around the primary jet core, possible originating from the separation region which is detailed in the schematic provided in Figure 14a. Proceeding further into the flowfield, the jet boundary region can be observed (lower-right image in Figure 14 b).
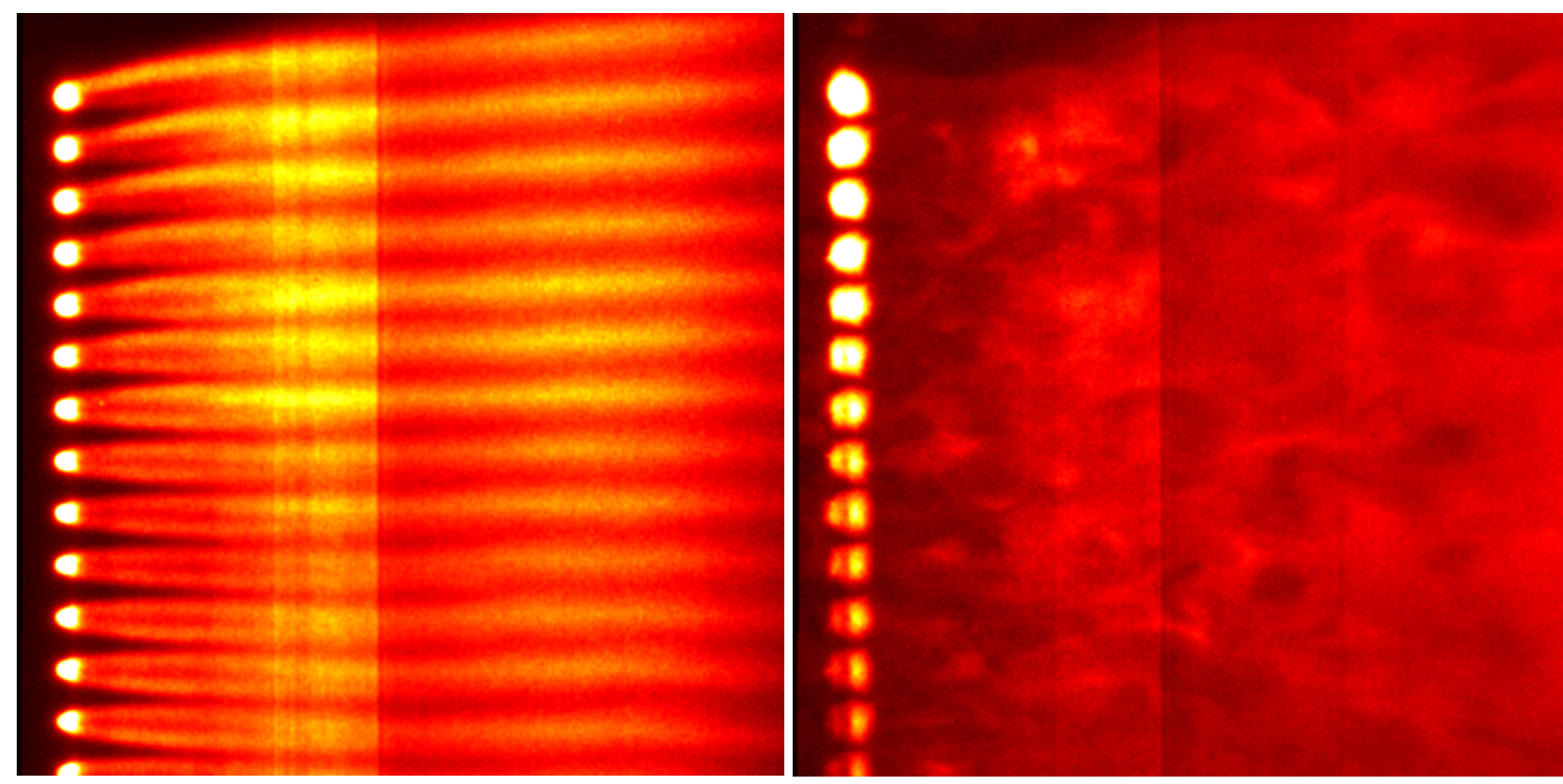

Figure 15. Runs 7 and 13 with a flow rate of $\sim 3$ slpm (left) and Runs 3 and 16 with a flow rate of $\sim 50$ slpm (right). The image contrast has been adjusted to demonstrate NO gas propagating in front of jets. 


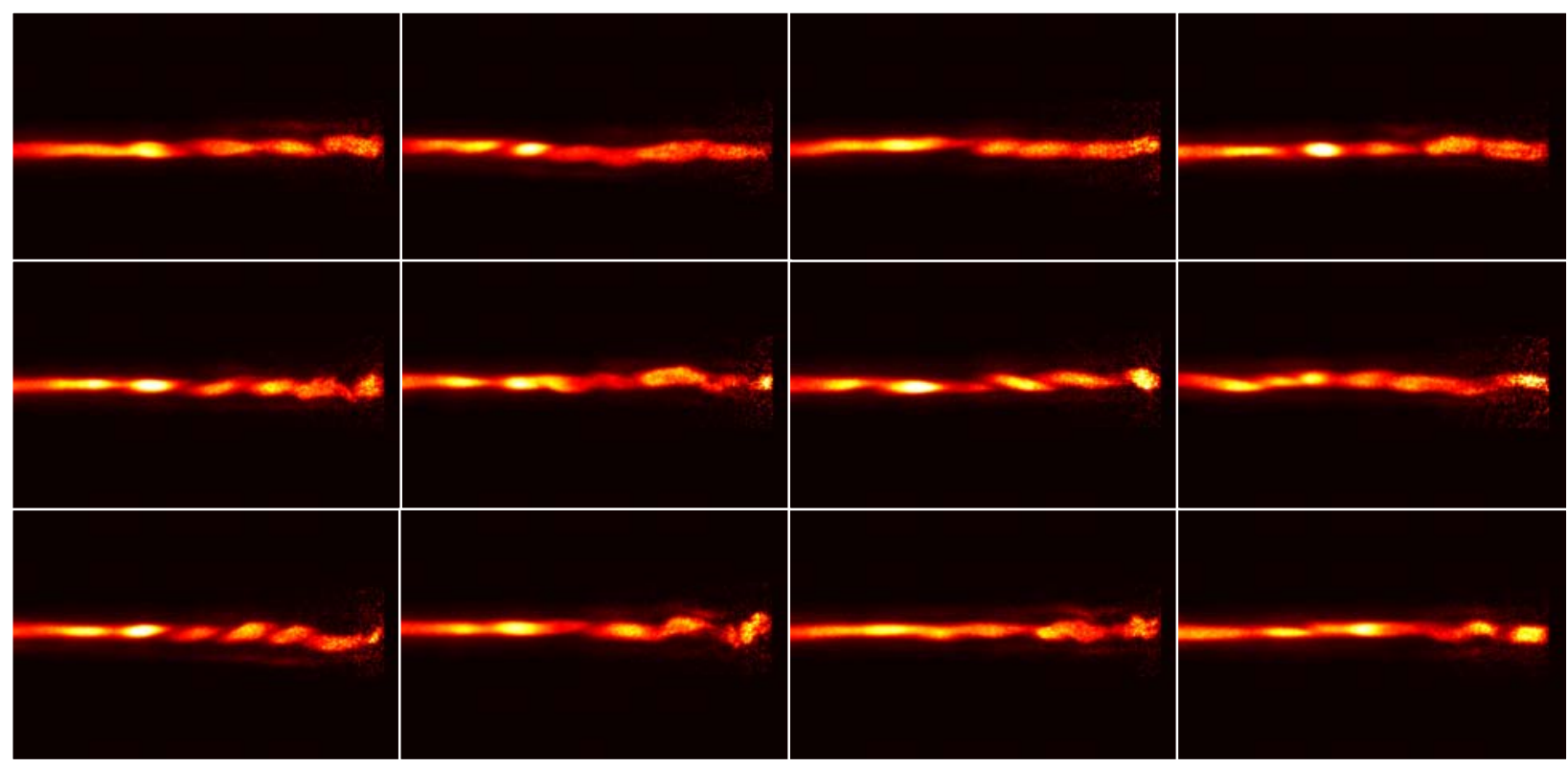

Figure 16. Run 17 with a flow rate of $\sim 0.61$ slpm (corresponding to Run 32 in Ref. 2 . PLIF images taken 0.15-inches off-body. Image set represents 12 consecutive images showing consistent corkscrew structure.

One of the more problematic issues encountered in this experiment was the shot-to-shot variation of laser intensity across the visualization plane. The cause of this was twofold; first, while care was taken to mitigate mechanical disturbance throughout the laser mounting system, slight intensity variations were a consequence of imparted tunnel vibration. Second, the laser system will inherently have shot-to-shot variation in photon intensity throughout the beam, which is especially apparent when the beam is expanded into a laser sheet for use in the PLIF technique. To rectify this issue, application of a higher power laser beam would allow for greater sheet expansion while producing a higher photon flux. However, by appropriately processing of the acquired PLIF images, a substantial amount of qualitative flow information can be extracted.

\section{Conclusions}

Application of planar laser-induced fluorescence (PLIF) imaging has been utilized to examine a perturbed boundary layer flow on a $1 / 3$ scale Hyper-X forebody model through the implementation of active blowing gas jets. The experiment was performed in NASA Langley Research Center's 31-inch Mach 10 wind tunnel. The target test conditions were selected to simulate conditions examined in a previous experiment where phosphor-thermography images were acquired detailing surface heating along the compression ramps of the Hyper-X forebody model. Two particular blowing trip configurations were considered: (1) a single row of 17 0.02-inch diameter holes and (2) a single 0.01-inch-diameter-hole trip. By seeding nitric oxide gas through the blowing gas jets and exciting planar slices of this gas at various off-body positions, characterization of the boundary layer profile was made. Comparisons were made between the acquired PLIF images and phosphor-thermography results to highlight similarities, and in some cases, discrepancies between boundary layer flow structures and increased surface heating regions. It was determined that in many cases, the flow structures observed at particular points off of the model body and the global surface heating measurements obtained though the phosphor thermography measurement did not entirely correlate to one another. It has been shown that the PLIF technique adds a significant flow visualization capability to the existing hypersonic boundary layer measurement methods.

Additionally, this experiment has provided insight concerning the separation region immediately upstream of the blowing jets was also provided based upon observation and analysis of both the PLIF images and heat transfer profiles. The acquired PLIF images also showed an apparent relation between the instabilities produced by the underexpanded jet at the transitional and turbulent nature of the corresponding downstream flow. 


\section{Acknowledgements}

We wish to acknowledge the contribution to this project from the NASA Langley Research Center 31-Inch Mach 10 Air Tunnel technicians and engineering, including Kevin Hollingsworth, Paul Tuck, Tony Robbins, Henry Fitzgerald and Johnny Ellis. Thanks also to Rich Schwartz and Andrew McCrea from ATK, Hampton, Virginia for additional assistance with computer visualizations. This work was supported by the NASA Fundamental Aeronautics Hypersonics Program.

\section{References}

${ }^{1}$ Berry, S.A., DiFulvio, M., and Kowalkowski, M.K., "Forced Boundary-Layer Transition on X-43 (Hyper-X) in NASA LaRC 20Inch Mach 6 Air Tunnel." NASA TM-2000-210316, August 2000.

2 Berry, S.A., Nowak, R.J., and Horvath, T.J., "Boundary Layer Control for Hypersonic Airbreathing Vehicles." AIAA 20042246, 34th AIAA Fluid Dynamics Conference and Exhibit, June 28 - July 1, 2004.

${ }^{3}$ Berry, S.A., Auslender, A.H., Dilley, A.D., and Calleja, J.F., "Hypersonic Boundary-Layer Trip Development for Hyper-X." Journal of Spacecraft and Rockets, 38, No. 6, November-December 2001.

${ }^{4}$ Berry, S.A., and Nowak, R.J., "A Comparison of Active and Passive Methods of Control of Hypersonic Boundary Layers on Airbreathing Configurations." JANNAF 27 th Airbreathing Propulsion Subcommittee Meeting, Colorado Springs, CO. 1-5 December 2003.

${ }^{5}$ Berrry, S.A., DiFulvio, M., and Kowalkowski, M.K., "Forced Boundary-Layer Transition on X-43 (Hyper-X) in NASA LaRC 31-Inch Mach 10 Air Tunnel." NASA TM-2000-210315, August 2000.

${ }^{6}$ Merski, R.N., "Global Aeroheating Wind-Tunnel Measurements Using Improved Two-Color Phosphor Thermography Method," Journal of Spacecraft and Rockets, 36, No. 2, March-April 1999.

${ }^{7}$ Stone, D.R. and Cary Jr., A.M., "Discrete Sonic Jets Used as Boundary-Layer Trips at Mach Numbers of 6 and 8.5." NASA TN-D-6802, July 1972.

${ }^{8}$ Danehy, P.M., Bathel, B.F., Inman, J.A., Alderfer, D.W., and Jones, S.B., "Stereoscopic Imaging in Hypersonic Flows using Planar Laser-Induced Fluorescence.” AIAA 2008-4267, AIAA Fluid Dynamic Conference, Seattle, WA, June 2008.

${ }^{9}$ Micol, J.R. "Langley Aerothermodynamic Facilities Complex: Enhancements and Testing Capabilities," AIAA 98-0147, 36th AIAA Aerospace Sciences Meeting \& Exhibit, January 12-15, Reno, NV, 1998.

10 Sorzano Sanchez, C.O., Thevenaz, P., and Unser M., "Elastic Registration of Biological Images Using Vector-Spline Regularization." IEEE Transactions on Biomedical Engineering, 52, No. 4, pp. 652-663, April 2005.

${ }^{11}$ Danehy, P. M., Wilkes, J.A., Brauckmann, G., Alderfer, D. W., Jones, S. B., and Patry, D., "Visualization of a Capsule Entry Vehicle Reaction-Control System (RCS) Thruster." AIAA 2006-1532, 44th AIAA Aerospace Sciences Meeting and Exhibit, Reno, Nevada, January 9-12, 2006.

12 (Wilkes) Inman, J. A., Danehy, P. M., Alderfer, D. W., Buck, G. M., McCrea, A., and Schwartz, R. J., "PLIF Imaging of Capsule RCS Jets, Shear Layers, and Simulated Forebody Ablation" AIAA Paper 2008-0248, 46th AIAA Aerospace Sciences Meeting and Exhibit, Reno, Nevada, January 7-10, 2008.

13 Wilkes, J. A., Alderfer, D. W., Jones, S. B. and Danehy, P. M., "Portable Fluorescence Imaging System for Hypersonic Flow Facilities," JANNAF Interagency Propulsion Committee Meeting, Colorado Springs, Colorado, December 2003.

${ }^{14}$ Schwartz, R. J., "ViDI: Virtual Diagnostics Interface Volume 1-The Future of Wind Tunnel Testing." Contractor Report NASA/CR-2003-212667, December 2003.

15 Alderfer, D.W., Danehy, P.M., (Wilkes) Inman, J.A., Berger, K.T., Buck, G.M., and Schwartz, R. J., "Fluorescence Visualization of Hypersonic Flow Over Rapid Prototype Wind-Tunnel Models." AIAA 2007-1063, 45th AIAA Aerospace Sciences Meeting and Exhibit, Reno, Nevada, January 8-11, 2007.

${ }^{16}$ Fay, J.A. and Ridell, F.R., "Theory of Stagnation Point Heat Transfer in Dissociated Air," Journal of Aerospace Sciences, 25, No. 2, pp. 73-85, 1958.

${ }^{17}$ Inman, J. A., Danehy, P. M., Nowak, R. J., Alderfer, D.W., "Identification of Instability Modes of Transition in Underexpanded Jets,” AIAA 2008-4389, 38th AIAA Fluid Dynamics Conference, Seattle, WA, June 23-26, 2008. 